\title{
Targeting interleukin-1 for reversing fat browning and muscle wasting in infantile nephropathic cystinosis
}

\author{
Wai W Cheung ${ }^{1 *}$, Sheng $\mathrm{Hao}^{2 *}$, Ronghao Zheng ${ }^{3}$, Zhen Wang ${ }^{4}$, Alex Gonzalez ${ }^{1}$, \\ Ping Zhou ${ }^{5}$, Hal M Hoffman ${ }^{6}$, Robert $\mathrm{H} \mathrm{Mak}^{1}$ \\ ${ }^{1}$ Pediatric Nephrology, Rady Children's Hospital San Diego, University of California, San Diego, La Jolla, USA \\ 2 Department of Nephrology and Rheumatology, Shanghai Children's Hospital, \\ Shanghai Jiao Tong University, Shanghai, China \\ ${ }^{3}$ Department of Pediatric Nephrology, Rheumatology, and Immunology, Maternal and Child Health Hospital \\ of Hubei Province, Tongji Medical College, Huazhong University of Science and Technology, Wuhan, China \\ ${ }^{4}$ Department of Pediatrics, Shanghai General Hospital, Shanghai Jiao Tong University, Shanghai, China \\ ${ }^{5}$ Department of Pediatrics, the Second Affiliated Hospital of Harbin Medical University, Harbin, China \\ ${ }^{6}$ Department of Pediatrics, University of California, San Diego, La Jolla, USA \\ * These authors contributed equally to this work. \\ Correspondence: \\ Robert H Mak \\ Division of Pediatric Nephrology \\ Department of Pediatrics \\ University of California, San Diego \\ 9500 Gilman Drive, MC0831, La Jolla, California 92093-0831 \\ P: 858-822-6717 \\ F: 858-822-6776 \\ E-mail: romak@health.ucsd.edu
}

word count: 3,916 


\section{ABSTRACT}

Background: Ctns $^{-/}$mice, a mouse model of infantile nephropathic cystinosis, exhibit hypermetabolism with adipose tissue browning and profound muscle wasting. Inflammatory cytokines such as IL-1 trigger inflammatory cascades and play an important role in the pathogenesis of cachexia. Anakinra is an FDAapproved IL-1 receptor antagonist that blocks IL-1 signaling and may provide targeted novel therapy.

Methods: $\mathrm{Ctns}^{-/}$mice were bred to $1 / 6^{\%}$ and $I / 1 \beta^{-/}$mice. Ctns ${ }^{-/}$mice and wild type control were treated with anakinra (2.5 mg.kg.day, IP) or saline as vehicle for 6 weeks. We quantitated total fat mass and studied expression of molecules regulating adipose tissue browning. We measured gastrocnemius weight, total lean mass content, muscle function (grip strength and rotarod activity), muscle fiber size, muscle fatty infiltration and expression of molecules regulating muscle metabolism. We also evaluated the effects of anakinra on the muscle transcriptome.

Results: II-1 $\beta$ deficiency or treatment with anakinra normalized food intake and weight gain, fat and lean mass content, metabolic rate and muscle function in $\mathrm{Ctns}^{-/}$mice. Anakinra also diminished molecular perturbations of energy homeostasis in adipose tissue and muscle, specifically, aberrant expression of beige adipose cell biomarkers (UCP-1, CD137, Tmem26 and Tbx1) and molecules implicated in adipocyte tissue browning (Cox2/Pgf2 $\alpha$, Tlr2, Myd88 and Traf6) in inguinal white adipose tissue in Ctns ${ }^{\%}$ mice. Moreover, anakinra normalized gastrocnemius weight and fiber size as well as attenuated muscle fat infiltration in $\mathrm{Ctns}^{\%}$ - mice. This was accompanied by correction of the increased muscle wasting signaling pathways (increased protein content of ERK1/2, JNK, p38 MAPK and NF-KB p65 and gene expression of Atrogin-1 and Myostatin) and the decreased myogenesis process (decreased gene expression of MyoD and Myogenin) in gastrocnemius of $\mathrm{Ctns}^{-/}$mice. Finally, anakinra normalized or attenuated 12 of those top 20 differentially expressed muscle genes in $\mathrm{Ctns}^{-/}$ mice.

Conclusions: Anakinra attenuates adipose tissue browning and muscle wasting in Ctns ${ }^{-/}$mice. IL-1 receptor blockade may represent a novel targeted treatment for cachexia in patients with infantile nephropathic cystinosis.

Key words: infantile nephropathic cystinosis, IL-1, cachexia, adipose tissue browning, muscle wasting 


\section{INTRODUCTION}

Cystinosis is a multisystem genetic disorder characterized by the accumulation of cystine in different tissues and organs. Infantile nephropathic cystinosis (INC) is the most common and severe form of cystinosis. ${ }^{3}$ Patients suffering from INC exhibit signs and symptoms of renal Fanconi syndrome and chronic kidney disease in early childhood. ${ }^{4}$ Metabolic abnormalities such as cachexia are common complications in patients with INC, which are associated with poor quality of life and mortality and for which there is no current therapy. ${ }^{3,4}$ Increased expression of inflammatory cytokines, including IL-1 $\beta$, has been implicated in the etiology of cachexia and muscle wasting associated with different disease processes. Consistent with this finding, patients with cystinosis had higher concentrations of circulating IL-1 $\beta$ compared with controls. ${ }^{6}$ PBMCs from patients with cystinosis revealed a significant increase in IL-1 $\beta$ transcript levels compared with controls. ${ }^{6}$ Recent evidence in preclinical models suggests that blockade of IL-1 signaling may be a logical therapeutic target for chronic disease-associated muscle wasting since IL-1 $\beta$ activates NF- $\kappa \beta$ signaling and induces expression of IL- 6 and atrogin-1 in C2C12 myocytes. ${ }^{7,8}$ Anakinra is an IL-1 receptor antagonist which blocks both IL-1 $\alpha$ and IL-1.$^{10}$ Duchene muscular dystrophy (DMD) is an X-linked muscle disease characterized by muscle inflammation that is associated with an increased circulating serum levels of IL-1 $\beta$. Subcutaneous administration of anakinra normalized muscle function in a mouse model of DMD. ${ }^{11}$ Similarly, serum IL-1 $\beta$ is elevated in hemodialysis patients and a 4-week treatment with anakinra was shown to be safe in these patients while significantly reducing markers of systemic inflammation such as CRP and IL-6. ${ }^{12}$ In this study, we investigated $\mathrm{Ctns}^{-/}$mice as an established model of INC in which we have previously shown hypermetabolism with adipose tissue browning and profound muscle wasting by 12 months of age. ${ }^{5}$ We explored the role of inflammatory cytokines in $\mathrm{Ctns}^{-}$mice, and evaluated the efficacy of IL-1 targeted therapy on adipose tissue browning and muscle wasting. Anakinra was FDA-approved for the treatment of rheumatoid arthritis in 2001 and is safe and an effective therapeutic option in a variety of diseases including diseases involving muscle. It may be repurposed as novel therapy for cachexia in INC.

\section{METHODS}

\section{Study design}

All animal work was conducted in compliance and approved by the Institutional Animal Care and Use Committee (IACUC) at the University of California, San Diego. Wild-type (WT), $116 \%$ and $111 \beta^{\%}, \mathrm{Ctns}^{\%}$, Ctns ${ }^{-}$ $116^{\%}$ and $\mathrm{Ctns}^{\%} \% 1 \beta^{\%}$ mice were on the same $\mathrm{c57 \textrm {BL } / 6}$ genetic background. Ctns ${ }^{\%}$ mice were kindly provided

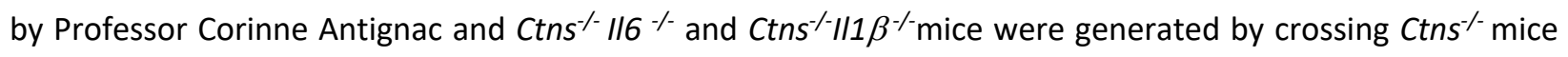
with $116^{\%}$ and $1 / 1 \beta^{\%}$ mice, respectively. Twelve-month old male mice were used for all of the following 5 studies. Study 1 - We evaluated the gastrocnemius muscle IL-1 $\beta$ and IL-6 mRNA and protein content in $\mathrm{Ctns}^{-/}$ 
mice and WT mice. Results were presented in Figure 1, A to D. Study 2 - We evaluated the effects of genetic deletion of IL-1 $\beta$ and IL- 6 in Ctns ${ }^{-/}$mice. We compared ad libitum food intake and weight change in WT, Ctns 1, Ctns ${ }^{\%} 116 \%$ and Ctns ${ }^{\%} / 11 \beta^{\%}$ mice. The study period was 6 weeks. Results were shown in Figure 1, E \& F. Study 3 - We evaluated the beneficial effects of genetic deletion of IL-1 $\beta$ and IL-6 in Ctns $^{-/}$mice beyond nutritional effects by employing a pair-feeding strategy. The study period was 6 weeks. Ctns $\%$ mice were fed

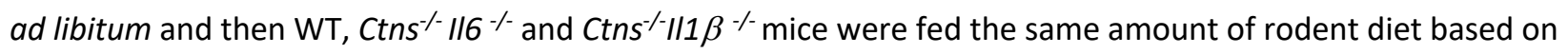
the recorded food intake of $\mathrm{Ctns}^{-/}$mice. Results were shown in Figure $1, \mathrm{G}$ to N. Study 4 - We evaluated the effects of anakinra in $\mathrm{Ctns}^{-/}$mice. WT and $\mathrm{Ctns}^{-/}$mice were given anakinra ( $2.5 \mathrm{mg} / \mathrm{kg}$.day, IP) or vehicle (normal saline), respectively. The study period was 6 weeks. All mice were fed ad libitum. We compared food intake and weight change in all groups of mice. Results were shown in Figure 2, A \& B. Study 5 - We evaluated the metabolic effects of anakinra in $\mathrm{Ctns}^{-}$mice beyond nutritional stimulation by employing a pair-feeding strategy. WT and $\mathrm{Ctns}^{-/}$mice were given anakinra (2.5 mg/kg.day, IP) or vehicle (normal saline), respectively. The study period was 6 weeks. Vehicle-treated $\mathrm{Ctns}^{-/}$mice were fed ad libitum while all other groups of mice were fed the same amount of rodent diet based on the recorded food intake of vehicle-treated $\mathrm{Ctns}^{-/}$mice. Results were shown in Figure 2, E to G \& Figures, 3 to 6 as well as Supplemental Figure $1 \& 2$.

\section{Serum and blood chemistry}

BUN and serum concentration of bicarbonate was measured (Supplemental Table 1S). Serum creatinine were analyzed by LC-MS/MS method. ${ }^{13}$

\section{Body composition, metabolic rate and in vivo muscle function}

Body composition (for lean and fat content) of mice were measured by quantitative magnetic resonance analysis (EchoMRI-100 ${ }^{\mathrm{TM}}$, Echo Medical System) twice, prior to the initiation of the study as well as at the end of the study and change of total body fat and lean mass in individual mouse was calculated. Twenty-four-hour metabolic rate $\left(\mathrm{VO}_{2}\right)$ of mice were measured using Oxymax indirect calorimetry (Columbus Instrument) at the end of the study. At the end of the study, muscle function (grip strength and rotarod activity) in mice was assessed using a grip strength meter (Model 47106, UGO Basile) and rotarod performance tool (model RRF/SP, Accuscan Instrument), respectively. ${ }^{5,14}$

\section{Protein assay for muscle and adipose tissue}

Mice were sacrificed at the end of the study and gastrocnemius muscle, inguinal white adipose tissue (WAT) and intercapsular brown adipose tissue (BAT) were dissected and processed in a homogenizer tube (USA Scientific, catalog 1420-9600) containing ceramic beads (Omni International, catalog 19-646) using a Bead Mill Homogenizer (Omni International). Protein concentration of tissue homogenate was assayed using Pierce BAC Protein Assay Kit (Thermo Scientific, catalog 23227). Uncoupling protein (UCP) protein content as well as adenosine triphosphate (ATP) concentration in adipose tissue and muscle homogenates were assayed. Protein 
concentration of phospho-ERK 1/2 (Thr202/Ty2r204) and total ERK 1/2, phospho-JNK (The183/Tyr185) and Total JNK, phospho-p38 MAPK (Thr180/Tyr182) and total p38 MAPK, NF-KB p65 (phospho-Ser536) and total NF-KB p65 as well as IL-6 and IL-1 $\beta$ in muscle homogenates were assayed (Supplemental Table 1S).

\section{Gastrocnemius wet weight, fiber size and fatty infiltration}

Left gastrocnemius muscle of mice was dissected at the end of the study. Wet weight of left gastrocnemius muscle was recorded. Subsequently, we measured muscle fiber cross-sectional area of gastrocnemius muscle. using ImageJ software (https://rsbweb.nih.gob/ij/)..$^{5,14}$ In addition, portion of dissected right gastrocnemius muscle samples were incubated with Oil Red O (Oil Red O Solution, catalog number 01391-250 ml, Sigma Aldrich). Detailed procedures for Oil Red O staining were in accordance with published protocol. ${ }^{15} \mathrm{We}$ followed a recently established protocol to quantify muscle fat infiltration. Acquisition and quantification of images were analyzed using ImageJ software. ${ }^{16}$

\section{Muscle RNAseq analysis}

Previously, we performed RNAseq analysis on gastrocnemius muscle mRNA in 12-month old $\mathrm{Ctns}^{-/-}$mice versus age-appropriate WT mice. ${ }^{14}$ Detailed procedures for mRNA extraction, purification and subsequent construction of cDNA libraries as well as analysis of gene expression was published. We then performed Ingenuity Pathway Analysis enrichment tests for those differentially expressed muscle genes in $\mathrm{Ctns}^{-/}$mice versus WT mice, focusing on pathways related to energy metabolism, skeletal and muscle system development and function, and organismal injury and abnormalities. We identified the top 20 differentially expressed muscle genes in $\mathrm{Ctns}^{-/}$versus WT mice. ${ }^{14}$ In this study, we performed qPCR analysis for those top 20 differentially expressed gastrocnemius muscle genes in the different experimental groups.

\section{Quantitative real-time PCR}

Total RNA from adipose and gastrocnemius muscle samples were isolated using TriZol (Life Technology) and reverse-transcribed with SuperScript III Reverse Transcriptase (Invitrogen). Quantitative real-time RT-PCR of target genes were performed using KAPA SYBR FAST qPCR kit (KAPA Biosystems). Expression levels were calculated according to the relative $2^{-\Delta \Delta C t}$ method. ${ }^{14}$ All primers are listed (Supplemental Table 2S).

\section{Statistical analysis}

Continuous variables are expressed as mean \pm S.E.M. We assessed the statistical significance of differences between groups using two-sample t-tests. All tests were two-sided. A $p$ value less than 0.05 was considered significant. Statistical analyses were performed using SPSS software version 16.0 for Macintosh. \# represents a statistically significant $(p<0.05)$ difference from WT control mice. Bars with specific $p$ values between groups represents a statistical significance from $\mathrm{Ctns}^{-/}$control mice. 


\section{RESULTS}

Increased muscle IL-6 and IL-1 $\beta$ mRNA and protein content in $\mathrm{Ctns}^{-/-}$mice

We chose to study $\mathrm{Ctns}^{\%}$ - mice at 12 months of age based on our previous data demonstrating a significant cachexia phenotype. As expected, all of the mice were uremic and had elevated creatine, but normal bicarbonate levels serum (Supplemental Table 3S). In order to investigate the role of inflammatory cytokines in cachexia and muscle wasting, we measured gastrocnemius muscle mRNA and protein content of IL-6 and IL$1 \beta$ in 12-month old $\mathrm{Ctns}^{-/}$mice and age-matched WT controls. Gastrocnemius mRNA and protein content of both IL-6 and IL-1 $\beta$ were significantly elevated in $\mathrm{Ctns}^{-/-}$mice relative to WT mice (Figure 1, A-D).

\section{Genetic deletion of IL-1 $\beta$ normalizes cachexia in $\mathrm{Ctns}^{-/}$mice}

We then employed a genetic approach to determine whether IL-6 and IL-1 $\beta$ deficiency would affect the cachexia phenotype in $\mathrm{Ctns}^{-/}$mice. Twelve-month old $\mathrm{Ctns}^{-/}, \mathrm{Ctns}^{-/ 116^{-/}}$and $\mathrm{Ctns}^{-/} / 11 \beta^{-\%}$ mice were all uremic and had elevated creatinine levels but normal bicarbonate levels (Supplemental Table 4S). All mice were fed ad libitum for 6 weeks and average daily food intake as well as final weight gain of the mice were recorded. We showed that genetic deletion of $I / 1 \beta$ completely corrected anorexia and normalized weight gain in $\mathrm{Ctns}^{-/}$ mice whereas deletion of $1 / 6$ only partially rescued the phenotype in $\mathrm{Ctns}^{-/}$mice (Figure 1, E\&F). Furthermore, to investigate the potential metabolic effects of genetic deficiency of $/ / 1 \beta$ and $/ / 6$ beyond its nutritional effects, we employed a pair-feeding strategy so $\mathrm{Ctns}^{-/}$mice were fed ad libitum and then all other groups of mice were fed the same amount of rodent diet based on the recorded food intake of the $\mathrm{Ctns}^{-/}$group (Figure 1G). Despite receiving the same amount of total calorie intake as other groups of mice, cardinal features of cachexia phenotype such as decreased weight gain, decreased fat mass content and hypermetabolism (manifested as elevated oxygen consumption), decreased lean mass content and gastrocnemius weight as well as reduced muscle function (decreased rotarod and grip strength) were still prominent in $\mathrm{Ctns}^{\%}$ mice (Figure $\mathbf{1}, \mathbf{H}$ to $\mathbf{N}$ ). Importantly, parameters of cachexia phenotype were normalized relative to WT mice or significantly improved

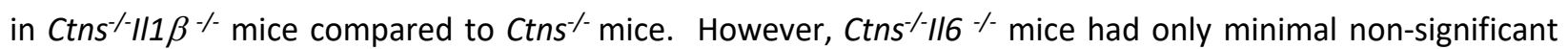
improvement relative to $\mathrm{Ctns}^{\%}$ - mice.

\section{Anakinra attenuates cachexia and muscle wasting in $\mathrm{Ctns}^{\%}$ mice}

In order to better model a real-world approach in patients, we used a pharmacological approach to test the effects of IL-1 $\beta$ blockade in pre-established INC-associated cachexia and muscle wasting. Anakinra, an IL-1 receptor antagonist that blocks both IL-1 $\alpha$ and IL-1 $\beta$ function was used to treat 12 -month old $\mathrm{Ctns}^{-/}$mice and WT controls for 6 weeks compared to vehicle. All mice were fed ad libitum. Food intake and weight gain was normalized in anakinra treated $\mathrm{Ctns}^{-/}$mice (Figure 2, A to B). We also investigated the beneficial effects of anakinra in $\mathrm{Ctns}^{-/}$mice beyond appetite stimulation and its consequent body weight gain through the utilization of a pair-feeding approach. Daily ad libitum food intake for $\mathrm{Ctns}^{-/}$mice treated with vehicle was 
recorded. Following that, anakinra treated $\mathrm{Ctns}^{-/}$mice as well as WT mice treated with anakinra or vehicle were food restricted such that the mice ate an equivalent amount of food as vehicle treated $\mathrm{Ctns}^{-/}$mice (Figure 2C). Serum and blood chemistry of mice were measured (Supplemental Table 5S). Anakinra normalized uremia, serum creatine, weight gain, and metabolic rate, and significantly improved or normalized fat and lean mass content, gastrocnemius weight, as well as muscle function (grip strength and rotarod activity) in $\mathrm{Ctns}^{\%}$ mice (Figure 2, D to J). Results of anakinra-treated $\mathrm{Ctns}^{-/}$mice were also compared to vehicle-treated $\mathrm{Ctns}^{-/}$mice. Anakinra normalized parameters of cachexia phenotype in $\mathrm{Ctns}^{\%}$ mice compared to vehicle-treated $\mathrm{Ctns}^{-/}$ mice.

\section{Anakinra normalizes energy homeostasis in adipose tissue and skeletal in $\mathrm{Ctns}^{-/-}$mice}

Adipose tissue (inguinal WAT and intercapsular BAT) and gastrocnemius protein content of UCPs was significantly increased in $\mathrm{Ctns}^{-/}$mice compared to WT mice (Figure 3, A, C \& E). In contrast, ATP content in adipose tissue and skeletal muscle was significantly decreased in $\mathrm{Ctns}^{\%}$ - mice compared to WT mice (Figure 3, B, D \& F). Anakinra normalized UCPs and ATP content of adipose tissue and muscle in Ctns ${ }^{\%-}$ mice.

Anakinra ameliorates browning of adipose tissue in $\mathrm{Ctns}^{\%}$ mice

Beige adipose cell surface markers (CD137, Tmem26 and Tbx1) mRNA levels were elevated in inguinal WAT in $\mathrm{Ctns}^{-1-}$ mice relative to WT mice (Figure 4, A to C). This was accompanied by an increased UCP-1 protein content in inguinal WAT shown in Figure 3A, which is a biomarker for beige adipocytes, and usually undetectable in WAT. Anakinra normalized the elevated protein content of inguinal WAT UCP-1 as well as increased mRNA expression of CD137, Tmen and Tbx1 in Ctns $\%$ mice (Figure 3A and Figure 4, A to C). Anakinra also ameliorated expression of important signature molecules implicated in WAT. Activation of the Cox $2 /$ Pgf $2 \alpha$ signaling pathway and chronic inflammation stimulate biogenesis of WAT browning. Significantly increased inguinal WAT mRNA expression of Cox2 and Pgf2 $\alpha$ was found in $\mathrm{Ctns}^{-/}$mice compared to WT mice (Figure 4, D and E). Moreover, inguinal WAT levels of toll-like receptor 2 (TIr2) as well as myeloid differentiation primary response 88 (MyD88) and tumor necrosis factor receptor-associated factor 6 (Traf6) were upregulated in $\mathrm{Ctns}^{-/}$mice compared to WT mice (Figure 4, F to $\mathbf{H}$ ) and anakinra significantly reduced inguinal WAT gene expression of Cox2/Pgf2 $\alpha$ as well as genes in the toll-like receptor pathway in $\mathrm{Ctns}^{-/-}$mice.

Anakinra improves muscle fiber size and attenuates muscle fat infiltration in $\mathrm{Ctns}^{-/}$mice.

We evaluated the effect of anakinra on skeletal muscle morphology in $\mathrm{Ctns}^{-/}$mice. Representative results of muscle sections are illustrated (Figure 5, A to D). Anakinra significantly improved average cross-sectional area of gastrocnemius fibers in $\mathrm{Ctns}^{-/}$mice (Figure 5E). We also evaluated fat infiltration of the gastrocnemius muscle in $\mathrm{Ctns}^{-/}$mice. Representative results of Oil Red O staining of muscle section are shown (Figure 5, $\mathrm{F}$ to I). We quantified intensity of muscle fat infiltration in mice and observed that anakinra attenuated muscle fat infiltration in $\mathrm{Ctns}^{-/}$mice (Figure 5J). 


\section{Anakinra attenuates signaling pathways implicated in muscle wasting in $\mathrm{Ctns}^{-/}$mice}

Muscle relative phospho-ERK 1/2 (Thr202/Tyr204), phospho-JNK (Thr183/Tyr185) and phospho-p38 MAPK protein content (Thr180/Tyr182) was significantly increased in Ctns $\%$ mice compared to WT mice (Figure 6, A-

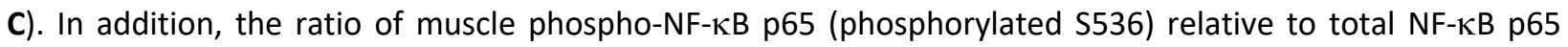
protein content was elevated in $\mathrm{Ctns}^{-/}$mice relative to WT mice (Figure 6, D). Importantly, anakinra attenuated or normalized phosphorylation of muscle ERK 1/2, JNK, p38 MAPK and NF-KB p65 protein content in Ctns ${ }^{-/}$ mice. Administration of anakinra improved muscle regeneration and myogenesis by decreasing mRNA expression of negative regulators of skeletal muscle mass (Atrogin-1 and Myostatin) (Figure 6, E and F) as well as increasing muscle mRNA expression of pro-myogenic factors (MyoD and Myogenin) in Ctns ${ }^{-/}$mice (Figure 6, G and $\mathbf{H})$.

\section{Molecular mechanism by RNAseq analysis}

Recently, we profiled differential expression of gastrocnemius mRNA between 12-month old $\mathrm{Ctns}^{-/}$mice and WT mice using RNAseq analysis. ${ }^{14}$ Ingenuity Pathway Analysis enrichment tests identified the top 20 differentially expressed muscle genes in $\mathrm{Ctns}^{-/}$mice versus WT mice. The top 15 up-regulated genes were Ankdr2, Csrp3, Cyfip2, Fhl1, Ly6a, Mup1, Myl2, Myl3, NIrc3, Sell, Sln, Spp1, Tnnc1, Tnni1, and Tpm3 whereas the top 5 down-regulated genes were Atf3, Cidea, Fos, Sncg and Tbc1d1 in Ctns ${ }^{-1}$ mice relative to WT mice. We performed qPCR analysis for those top 20 differentially expressed muscle genes in the present study. Importantly, anakinra significantly reduced (Ankdr2, Csrp3, Cyfip2, Fhl1, Ly6a, Nlrc3, Tnnc1, and Tpm3) and significantly increased (Atf3, Cidea, Sncg, and Tbc1d1) muscle gene expression in $\mathrm{Ctns}^{-/}$mice relative to WT mice (Supplemental Figure 1 \& 2). Functional significance of each of these 12 genes is listed (Table 1). Nonsignificant changes were observed in Mup1, Myl2, Myl3, Sell, Sln, Spp1, Tnni1 and Fos.

\section{DISCUSSION}

Previously, we described cachexia characterized by adipose tissue browning and muscle wasting in Ctns ${ }^{-/}$mice, an established mouse model of INC, but the etiology was not clear. Increased expression of inflammatory cytokines such as IL-6 and IL-1 have been implicated in the etiology of cachexia and muscle. Our studies using specific cytokine deficient mice and IL-1 targeted therapy suggest that IL-1 is a critical cytokine in INCassociated cachexia. The novel findings of this study are (i) Anakinra corrected anorexia, normalized weight gain, and attenuated hypermetabolism. (ii) Anakinra attenuated the expression of markers of adipose tissue browning as well as perturbed energy expenditure and thermogenesis. (iii) Anakinra attenuated muscle wasting, improved muscle function and corrected perturbations of aberrant expression of molecules implicated in muscle wasting. Together, our results suggest that anakinra may be an effective targeted treatment approach for cachexia in patients with INC. 
IL-1 $\beta$ suppresses food intake, activates energy metabolism and reduces weight gain in experimental animals., ${ }^{9,17}$ IL-1 $\beta$ signals through appetite-regulating neuropeptides, such as leptin resulting in appetite suppression. ${ }^{18}$ We have demonstrated that elevated circulating level of leptin through the activation of melanocortin receptor 4 induces CKD-associated cachexia. ${ }^{19}$ For this present study, we showed that skeletal muscle IL-1 $\beta$ level was significantly increased in $\mathrm{Ctns}^{\%}$ mice, and $\mathrm{Ctns}^{\%} / 11 \beta^{\%}$ mice had normalized parameters of cachexia phenotype relative to control mice (Figure 1). Furthermore, we showed that anakinra improved anorexia and normalized weight gain in $\mathrm{Ctns}^{-/}$mice relative to control mice (Figure 2). Our results further highlight the beneficial effects of anakinra beyond food stimulation and accompanied weight gain. In pair-fed studies in which anakinra-treated $\mathrm{Ctns}^{\%}$ mice and anakinra-treated WT mice were fed the same amount of food, cachexia was attenuated in anakinra-treated $\mathrm{Ctns}^{-1}$ mice relative to control mice (Figure 2).

The basal metabolic rate accounts for up to $80 \%$ of the daily calorie expenditure by individual. ${ }^{20}$ Skeletal muscle metabolism is a major determinant of resting energy expenditure. ${ }^{21,22}$ IL-1 $\beta$ increases basal metabolic rate (as represented by an increase in resting oxygen consumption) in a dose-dependent manner. ${ }^{23}$ In our study, anakinra normalized the increased 24-hr metabolic rate in $\mathrm{Ctns}^{-/}$mice (Figure $2 \mathrm{~J}$ ). Adipose tissue UCP1 expression is essential for adaptive adrenergic non-shivering thermogenesis and muscle UCP3 level controls body metabolism. ${ }^{24}$ The energy generated when dissipating the proton gradient via upregulation of UCPs is not used for cellular ATP production or other biochemical processes but instead to produce heat. ${ }^{25,26}$ We showed that anakinra normalized muscle and adipose tissue UCPs and ATP content in Ctns ${ }^{-}$mice (Figure 3). Blockade of IL-1 receptor signaling may also mitigate the metabolic dysfunction through leptin signaling. Infusion of leptin increased UCPs expression in skeletal muscle and adipose tissue..$^{27,28}$

Perhaps our most novel finding is that anakinra reduced adipose tissue browning in $\mathrm{Ctns}^{-/}$mice. New evidence suggests a maladaptive role of adipose tissue browning in the context of cachexia. ${ }^{29}$ Activation of adipose tissue browning is associated with profound energy expenditure and weight loss in cachectic patients. Browning of adipose tissue increases energy expenditure and takes place before skeletal muscle atrophy in murine models of cancer and CKD-associated muscle wasting. ${ }^{30,31}$ We previously demonstrated adipose tissue browning in $\mathrm{Ctns}^{-/}$mice (as evidenced by the detection of inguinal WAT UCP1 protein and increased expression of beige adipose cell markers CD137, Tmem and Tbx1). ${ }^{5,14}$ Cox2 is a downstream effector of $\beta$-adrenergic signaling and induces biogenesis of beige cells in WAT depots. ${ }^{32}$ We showed that anakinra normalized inguinal WAT Cox2, Pgf2 $\alpha$ as well as important inflammatory molecules (TIr2, MyD88 and Trap6) expression in Ctns ${ }^{-1-}$ mice and normalized key inflammatory molecules (TIr2 and MyD88) involved in adipose tissue browning in $\mathrm{Ctns}^{\%}$ mice (Figure 4). Recent data suggest that IL1 $\beta$ signaling mediates adipocyte browning via regulating of mitochondrial oxidative responses in both cultured human and animal adipocytes. ${ }^{33}$ 
We studied the impact of anakinra on muscle wasting in $\mathrm{Ctns}^{-/}$mice. Anakinra normalized lean mass content, gastrocnemius wet weight as well as muscle function in $\mathrm{Ctns}^{-/-}$mice relative to WT mice (Figure 2, $\mathbf{K}$ to $\mathbf{N})$. Furthermore, anakinra normalized average cross-sectional area as well as muscle fat infiltration of gastrocnemius muscle in $\mathrm{Ctns}^{-}$- mice (Figure 5, A-E). Muscle fat infiltration is a significant predictor of both muscle function and mobility function across a wide variety of comorbid conditions such as diabetes, spinal cord injury and kidney disease. ${ }^{34-36}$ Muscle adipose tissue may release pro-inflammatory cytokines within the muscle and impair the local muscle environment, impair blood flow or increase the rate of lipolysis within skeletal muscle resulting in an increased concentration of glucose within the skeletal muscle itself followed by insulin resistance. ${ }^{37,38}$

We investigated the impact of anakinra on signaling molecules that modulate muscle mass regulation in $\mathrm{Ctns}^{\%}$ mice. Systemic inflammation decreases muscle regeneration and increases muscle catabolism that eventually leads to muscle wasting in CKD. Inhibition of IL-1 reduced systemic inflammation (decreased serum CRP and IL-6) in CKD patients. ${ }^{12,39}$ A recent study also showed that blockade of IL-1 signaling significantly improved inflammatory status (a decrease in plasma concentration of IL-6 and TNF) and antioxidative properties in CKD patients. ${ }^{40}$ Systemic inflammation, as assessed by serum concentration of CRP, is a strong and independent risk factor for skeletal muscle wasting in CKD patients. ${ }^{41} \mathrm{IL}-1$ has been shown to stimulate the expression of catabolic genes. ${ }^{10,42,43}$ MAPK are a family of protein phosphorylating enzymes that regulate a diverse aspect of cellular responses including skeletal muscle regeneration and differentiation. ${ }^{44-46}$ Activation of the NF-KB signaling pathway leads to severe muscle wasting in mice. ${ }^{47}$ IL-1 $\alpha$ and IL-1 $\beta$, both isoforms of IL1 , stimulated catabolism in $\mathrm{C} 2 \mathrm{C} 12$ myotubes via activation of NF- $\mathrm{KB}$ signaling and atrogin-1 expression. ${ }^{7}$ Importantly, anakinra attenuated or normalized phosphorylation of muscle ERK 1/2, JNK, p38 MAPK and NF$\kappa \mathrm{B}$ p65 protein content in $\mathrm{Ctns}^{-/}$mice. In addition, anakinra improved muscle regeneration and myogenesis by decreasing mRNA expression of negative regulators of skeletal muscle mass (Atrogin-1 and Myostatin) as well as increasing muscle mRNA expression of pro-myogenic factors (MyoD and Myogenin) in $\mathrm{Ctns}^{-/}$mice (Figure 6). Our findings were in agreement with a recent report demonstrating that IL-1 $\beta$ administration inhibiting muscle mRNA expression of atrogin- $1 .^{9}$

Finally, we studied the impact of anakinra on muscle transcriptomics in $\mathrm{Ctns}^{-/-}$mice. We showed that anakinra normalized or attenuated muscle expression of 12 out of 20 top differentially expressed genes in Ctns /- mice. ${ }^{14}$ Detailed functional significance of each of these 12 differentiated expressed muscle genes is listed in Table 1. Anakinra normalized (Csrp3, Ly6a, Nlrc3, Tnnc1 and Tpm3, Atf3, Cidea, Sncg and Tbc1d1) and attenuated (Cyfip2 and Fhl1) muscle gene expression in $\mathrm{Ctns}^{-/-}$mice relative to WT mice (Supplemental figure 1 \& 2). Increased expression of Csrp3, Cyfip2, Fhl1, Nlcr3, Tnnc1 and Tpm3 have been implicated in muscle atrophy and muscle weakness. ${ }^{48-53}$ Increased expression of Ly6a has been associated with aberrant remodeling 
of the extracellular matrix during skeletal muscle regeneration. ${ }^{54}$ In addition, increased expression of Ankrd2 as well as decreased expression of Atf3 impairs muscle function and impairs survival of motor neuron and muscle innervation. ${ }^{55-57}$ Recent data also suggest that decreased expression of Cidea and Sncg are associated with higher energy expenditure as well as accelerated lipolysis of adipose tissue. ${ }^{58,59}$ Furthermore, decreased expression of Tbc1d1 impairs glucose transport in skeletal muscle and has been implicated in follistatin-induced muscle hypertrophy. ${ }^{60}$

\section{CONCLUSION}

We report that anakinra attenuates adipose tissue browning and muscle wasting in $\mathrm{Ctns}^{-/}$via multiple cellular mechanisms (Figure 7). IL-1 receptor antagonist may represent a novel targeted treatment for cachexia in patients with INC by attenuating adipose tissue browning and muscle wasting. 


\section{ACKNOWLEDGMENTS}

All authors of this manuscript certify that they complied with the ethical guidelines for authorship and publishing in the Journal of Cachexia, Sarcopenia and Muscle update 2017. ${ }^{61}$

\section{FUNDING}

Ping Zhou was supported by "Spring Sunlight Program" cooperative research project of Ministry of education (HLJ2019023) and Research Fund for Young \& Middle-Aged Innovative Science of the Second Affiliated Hospital of Harbin Medical University (CX2016-03). Robert Mak is funded by grants from the NIH: RO1 DK125811, R01 HD095547, U01 DK066143; California Institute of Regenerative Medicine: CLIN2-11478 and from the Cystinosis Research Foundation. Hal Hoffman is funded by NIH awards: R01 DK113592, R01 HL140898, R01 R01Al134030 and a UC collaborative grant.

\section{CONFLICTS OF INTEREST}

None 


\section{REFERENCES}

1. Town M, Jean G, Cherqui S, Attard M, Forestier L, Whitmore SA, et al. A novel gene encoding an integral membrane protein is mutated in nephropathic cystinosis. Nat Genet 1998;18:319-324.

2. Gahl WA, Thoene JG, Schneider JA. Cystinosis. N Engl J Med 2002;347:111-121.

3. Nesterova G, Gahl W. Nephropathic cystinosis: late complications of a multisystemic disease. Pediatr Nephrol 2008;23:863-878.

4. Theodoropoulos DS, Krasnewich D, Kaiser-Kupfer MI, Gahl WA. Classic nephropathic cystinosis as an adult disease. JAMA 1993;270:2200-2204.

5. Cheung W, Cherqui S, Ding W, Esparza W, Zhou P, Shao J, et al. Muscle wasting and adipose tissue browning in infantile nephropathic cystinosis. J Cachexia Sarcopenia Muscle 2016;7:152-164.

6. Prencipe G, Caiello I, Cherqui S, Whisenant T, Petrini S, Emma F, et al. Inflammasome activation by cystine crystals: Implications for the pathogenesis of cystinosis. J Am Soc Nephrol 2014;25:1163-1169.

7. Li W, Moylan JS, Chambers MA, Smith J, Reid MB: Interleukin-1 stimulates catabolism in C2C12 myotubes. Am J Physiol Cell Physiol 2009;297:C706-714.

8. Huang N, Kny M, Riediger F, Busch K, Schmidt S, Luft FC, et al. Deletion of Nlrp3 protects from inflammationinduced skeletal, muscle atrophy. Intensive Care Med Exp 2017;5:3, doi: 10.1186/s40635-016-0115-0.

9. Braun TP, Zhu X, Szumowski M, Scott GD, Grossberg AJ, Levasseur PR, et al. Central nervous system inflammation induces muscle atrophy via activation of the hypothalamic-pituitary-adrenal axis. J Exp Med 2011;208:2449-2463.

10. Dinarello CA. Overview of the IL-1 family in innate inflammation and acquired immunity. Immunol Rev 2018;281:8-27.

11. Klimek MEB, Sali A, Rayavarapu S, Van der Meulen JH, Nagaraju K. Effect of the IL-1 Receptor Antagonist Kineret on Disease Phenotype in $\mathrm{mdx}$ Mice. PLos One 2016;11:e0155944. doi: 10.1371/journal.pone.0155944.

12. Hung $A M$, Ellis $C D$, Shintani $A$, Booker C, Ikizler TA. IL-1 $\beta$ receptor antagonist reduces inflammation in hemodialysis patients. J Am Soc Nephrol 2011;22:437-442.

13. Young S, Struys E, Wood T. Quantification of creatinine and guanidinoacetate using GC-MS and LC-MS/MS for the detection of cerebral creatinine deficiency syndromes. Current Protocol in Human Genetics 2007;17.3.117.3.18.

14. Cheung W, Hao S, Wang Z, Ding W, Zheng R, Gonzalez A, et al. Vitamin D repletion ameliorates adipose tissue browning and muscle wasting in infantile nephropathic cystinosis. J Cachexia Sarcopenia Muscle 2020;11:120134.

15. Dubowitz V. Histological and histochemical stains and reactions. In Muscle biopsy. A practical approach 4th edn (eds Dubowitz V. et al.) (Elsevier, Amsterdam, 2013).

16. Mehlem A., Hagberg CE, Kuhl L, Eriksson U, Falkwvall A. Imaging of neutral lipids by oil red $O$ for analyzing the metabolic status in health and disease. Nat Protoc 2013;8:1149-1154.

17. Tocco-Bradley R, Georgieff M, Jones CT, Moldawer LL, Dinarello CA, Blackburn GL, et al. Changes in energy expenditure and fat metabolism in rats infused with interleukin-1. Eur J Clin Invest 1987;17:504-510.

18. Sachot C, Poole S, Luheshi GN. Circulating leptin mediates lipopolysaccharide-induced anorexia and fever in rats. J Physiol 2004;561(Pt 1):263-272.

19. Cheung W, Yu PX, Little BM, Cone RD, Marks DL, Mak RH. Role of leptin and melanocortin signaling in uremiaassociated cachexia. J Clin Invest 2005;115:1659-1665.

20. Eckel-Mahan K, Sassone-Corsi P. Metabolism and the Circadian Clock Converge. Physiol Rev 2013;93:107-135.

21. van den Berg SA, van Marken Lichtenbelt W, Willems van Dijk K, Schrauwen P. Skeletal muscle mitochondrial uncoupling, adaptive thermogenesis and energy expenditure. Curr Opin Clin Nutr Metab Care 2011:14:243249.

22. Zurlo F, Larson K, Bogardus C, Ravussin E. Skeletal muscle metabolism is a major determinant of resting energy expenditure. J Clin Invest 1990;86:1423-1427.

23. Dascombe MJ, Rothwell NJ, Sagay BO, Stock MJ. Pyrogenic and thermogenic effects of interleukin 1 beta in 
the rat. Am J Physiol 1989;256(1 Pt 1):E7-E11.

24. Rousset S, Alves-Guerra MC, Mozo J, Miroux B, Cassard-Doulcier AM, Bouillaud F, et al. The biology of mitochondrial uncoupling proteins. Diabetes 2004;53:S130-S135.

25. Argiles JM, Busquets S, Lopez-Sorian FJ. The role of uncoupling proteins in pathophysiological states. Biochem Biophy Res Commun 2002;293:1145-1152.

26. Sluse FE. Uncoupling proteins: molecular, functional, regulatory, physiological and pathological aspects. Adv Exp Med Biol 2012;942:137-156.

27. Rouru J, Cusin I, Zakrzewska KE, Jeanrenaud B, Rohner-Jeanrenaud F. Effects of intravenously infused leptin on insulin sensitivity and on the expression of uncoupling proteins in brown adipose tissue. Endocrinology 1999;140:3688-3692.

28. Tajima D, Masaki T, Hidaka S, Kakuma T, Sakata T, Yoshimatsu H. Acute central infusion of leptin modulates fatty acid mobilization by affecting lipolysis and mRNA expression for uncoupling proteins. Exp Biol Med (Maywood) 2005;230:200-206.

29. Kir S, White J, Kleiner S, Kazak L, Cohen P, Baracos VE, et al. Tumour-derived PTH related protein triggers adipose tissue browning and cancer cachexia. Nature 2014;513:100-104.

30. Petruzzelli M, Wagner EF. Mechanisms of metabolic dysfunction in cancer cachexia. Genes Dev 2016;30:489501.

31. Kir S, Komaba H, Garcia AP, Economopoulos KP, Liu W, Lanske B, et al. PTH/PTHrP receptor mediates cachexia in models of kidney failure and cancer. Cell Metab 2016;23:315-323.

32. Vegiopoulos A, Müller-Decker K, Strzoda D, Schmitt I, Chicheknitskiy E, Ostertag A, et al. Cyclooxygenase-2 controls energy homeostasis in mice by de novo recruitment of brown adipocytes. Science 2010;328:11581161.

33. Okla M, Zaher W, Alfayez M, Chung S. Inhibitory effects of Toll-like receptor 4, NLRP3 Inflammasome, and interleukin-1 $\beta$ on white adipocyte browning. Inflammation 2018;41:626-642.

34. Addison O, Marcus RL, LaStayo PC, Yuan AS. Intermuscular fat: A review of the consequences and causes. Int J Endocrino 2014;Article ID: 309570.

35. Goodpaster BH, Thaete FL, Kelley DE. Thigh adipose tissue distribution is associated with insulin resistance in obesity and in type 2 diabetes mellitus. Am J Clin Nutr 2000;71:885-892.

36. Cheema B, Abas H, Smith B, O'Sullivan AJ, Chan M, Patwardhan A, et al. Investigation of skeletal muscle quality and quality in end-stage renal disease. Nephrology (Carlton) 2010;15:454-463.

37. Beasley LE, Koster A, Newman AB, Javaid MK, Ferrucci L, Kritchevsky SB, et al. Inflammation and race and gender differences in computerized tomography-measured adipose depots. Obesity 2009;17:1062-1069.

38. Sam S, Haffner S, Davidson MH, D'Agonstino RB, Feinstein S, et al. Relation of abdominal fat depots to systemic markers of inflammation in type 2 diabetes. Diabetes Care 2009;32:932-937.

39. Nowak KL, Chonchol M, Ikizler TA, Farmer-Bailey H, Salas N, Chaudhry R, et al. IL-1 inhibition and vascular function in CKD. J Am Soc Nephrol 2017;28:971-980.

40. Hung AM, Tsuchida Y, Nowak KL, Sarkar S, Chonchol M, Whitfield V, et al. IL-1 inhibition and function of the HDL-containing fraction of plasma in patients with stages 3 to 5 CKD. Clin J Am Soc Nephrol 2019;14:702-711.

41. Deger SM, Hung AM, Gamboa JL, Siew ED, Ellis CD, Booker C, et al. Systemic inflammation is associated with exaggerated skeletal muscle protein catabolism in maintenance hemodialysis patients. $\mathrm{JCl}$ Insight 2017;2(22):e95185.

42. Londhe P, Guttridge DC. Inflammation induced loss of skeletal muscle. Bone 2014;80:131-142.

43. Ballak DB, Stienstra R, Tack CJ, Dinarello CA, van Diepen JA. IL-1 family members in the pathogenesis and treatment of metabolic disease: Focus on adipose tissue inflammation and insulin resistance. Cytokine 2015;75:280-290.

44. Geisler HWS, Shi H, Gerrard DE. MAPK pathway in skeletal muscle diseases. J Vet Anim Husb 2013;1:e104.

45. Zetser A, Gredinger E, Bengal E. p38 mitogen-activated protein kinase pathway promotes skeletal muscle differentiation. Participation of the Mef2c transcription factor. J Biol Chem 1999;274:5193-5200.

46. Wu Z, Woodring PJ, Bhakta KS, Tamura K, Wen F, Feramisco JR, et al. p38 and extracellular signal-regulated kinases regulate the myogenic program at multiple steps. Mol Cell Biol 2000;20:3951-3964. 
47. Cai D, Frantz JD, Tawa NE Jr, Melendez PA, Oh BC, Lidov HG, et al. IKKbeta/NF-kappaB activation causes severe muscle wasting in mice. Cell 2004;119:285-298.

48. Cui C, Han S, Tang S, He H, Shen X, Zhao J, et al. The autophagy regulatory molecules CSRP3 interacts with LC3 and protects against muscular dystrophy. Int J Mol Sci 2020;21(3):749.

49. Llano-Diex M, Gustafson AM, Olsson C, Goransson H, Larsson L. Muscle wasting and the temporal gene expression pattern in a novel rat intensive care unit model. BMC Genomics 2011;12:602.

50. Lee JY, Lori D, Wells DJ, Kemp PR. FHL1 activates myostatin signaling in skeletal muscle and promotes atrophy. FEBS Open Bio 2015;5:753-762.

51. Karki R, Malireddi RKS, Zhu Q, Kanneganti TD. NLRC3 regulates cellular proliferation and apoptosis to attenuate the development of colorectal cancer. Cell Cycle 2017;16:1243-1251.

52. Johnson JR, Chase PB, Ointo JR. Troponin through the looking-glass: emerging roles beyond regulation of straited muscle contraction. Oncotarget 2018;9:1461-1482.

53. Yuen M, Cooper ST, Marston SB, Nowak KJ, McNamara E, Mokbel N, Ilkovski B, et al. Muscle weakness in TPM3-myopathy is due to reduced Ca2+-sensitivity and impaired acto-myosin cross-bridge cycling in slow fibres. Hum Mol Genet 2015;24:6278-6292.

54. Kafadar KA, Yi L, Ahmad Y, So L, Rossi F, Pavlath GK. Sca-1 expression is required for efficient remodeling of the extracellular matrix during skeletal muscle regeneration. Dev Bio 2009;326:47-59.

55. Kemp TJ, Sadusky TJ, Saltisi F, Carey N, Moss J, Yang SY, et al. Identification of Ankrd2, a novel skeletal muscle gene coding for a stretch-response ankyrin-repeat protein. Genomics 2000;66:229-241.

56. Mohamed JS, Lopez MA, Cox GA, Boriek AM. Anisotropic regulation of Ankrd2 gene expression in skeletal muscle by mechanical stretch. FASEB J 2010;24:3330-3340.

57. Shijffers R, Zhang J, Matthews JC, Chen A, Tamrazian E, Babaniyi O, et al. Atf3 expression improves motor function in the ALS mouse model by promoting motor neuron survival and retaining muscle innervation. Proc Natl Acad Sci USA 2014;111:1622-1627.

58. Abreu-Vieira G, Fischer AW, Mattsson C, de Jong JM, Shabalina IG, Ryden M, et al. Cidea improves the metabolic profile through expansion of adipose tissue. Nat Commun 2015;6: 7433.

59. Shao $\mathrm{T}$, Song $\mathrm{P}$, Hua $\mathrm{H}$, Zhang $\mathrm{H}$, Sun $\mathrm{X}$, Kong $\mathrm{X}$, et al. Gamma synuclein is a novel Twist 1 target that promotes TGF- $\beta$-induced cancer cell migration and invasion. Cell Death Dis 2018;9:625.

60. Han X, Moller LLV, Groote ED, Bojsen-Moller KN, Davey J, Henriquez-Olguin CH, et al. Mechanisms involved in follistatin-induced hypertrophy and increase insulin action in skeletal muscle. J Cachexia Sarcopenia Muscle 2019;10:1241-1257.

61. von Haehling S, Morley JE, Coats AJS, Anker SD. Ethical guidelines for publishing in the Journal of Cachexia, Sarcopenia and Muscle: update 2017. J Cachexia Sarcopenia Muscle 2017;8:1081-1083. 
Table 1: Anakinra normalizes or attenuated expression of important muscle genes that have been implicated in muscle wasting in $\mathrm{Ctns}^{\%}$ mice. Previously, we studied differential expression of gastrocnemius mRNA between $\mathrm{Ctns}^{-/}$mice and WT mice using RNAseq analysis. ${ }^{14}$ We focus on pathways related to energy metabolism, skeletal and muscular system development and function, nervous system development and function as well as organismal injury and abnormalities. We performed qPCR analysis for top 20 differentially expressed muscle genes in the present study. Importantly, anakinra normalized (Ankdr2, Csrp3, Ly6a, Nlrc3, Tnnc1 and Tpm3, Atf3, Cidea, Sncg and Tbc1d1) and attenuated (Cyfip2 and Fhl1) muscle gene expression in $\mathrm{Ctns}^{-1-}$ mice relative to WT mice. Functional significance of each of these differentially expressed muscle genes is listed. DEG, differential expressed genes.

\begin{tabular}{|c|c|}
\hline Upregulated DEG & Functional significance $\&$ reference \\
\hline Ankrd2 & implicated in mechanical stretch of skeletal muscle 55,56 \\
\hline Csrp3 & associated with skeletal muscle dystrophy 48 \\
\hline Cyfip2 & associated with muscle wasting 49 \\
\hline Fhl1 & activates myostatin signaling and promotes atrophy in skeletal muscle 50 \\
\hline Ly6a & associated with remodeling of the extracellular matrix during skeletal muscle regeneration \\
\hline Nlrc3 & implicated in skeletal muscle wasting by inhibiting cell proliferation and promoting cell apo \\
\hline Tnnc1 & regulates straited muscle contraction 52 \\
\hline & implicated in cardiomyopathy pathogenesis and age-related skeletal muscle wasting 52 \\
\hline Tpm3 & promotes slow myofiber hypotrophy and associated with generalized muscle weakness ${ }^{53}$ \\
\hline Downregulated DEG & Functional significance \\
\hline Atf3 & impairs motor neuron survival and muscle innervation 57 \\
\hline Cidea & Increases metabolic rates, lipolysis in brown adipose tissue and higher core temperature 58 \\
\hline Sncg & Increases energy expenditure, particularly in brown and white adipose tissue 59 \\
\hline & associated with cancer cachexia through the TGF- $\beta$ signaling pathway 59 \\
\hline Tbc1d1 & Impairs glucose transport in skeletal muscle ${ }^{60}$ \\
\hline & associated with follistatin-induced muscle hyper \\
\hline
\end{tabular}




\section{Figure legends}

Figure 1: Increased muscle mRNA and protein content of IL- 6 and IL-1 $\beta$ in $\mathrm{Ctns}^{-/}$mice and genetic depletion of IL-1 $\beta$ provides a better rescue of cachexia in $\mathrm{Ctns}^{-/}$mice compared to IL-6 deficiency. Results of 3 different experiments were shown. For the $1^{\text {st }}$ study, we compared gene expression and protein content of IL- 6 and IL$1 \beta$ in gastrocnemius muscle in 12-month old $\mathrm{Ctns}^{-/}$and WT mice. Data were expressed as mean $\pm \mathrm{SEM}$. Results of $\mathrm{Ctns}^{-1}$ mice were compared to WT mice (A to D). For the $2^{\text {nd }}$ study, we compared the metabolic effects of genetic deletion of IL-6 and IL-1 $\beta$ in Ctns ${ }^{-/}$mice. Four groups of mice were included, i.e., Ctns ${ }^{-1}, \mathrm{Ctns}^{-/} \mathrm{II}^{-/}$and $\mathrm{Ctns}^{-} / 111 \beta^{-\%}$ as well as WT mice. All mice were 12 -month old and were fed ad libitum and the study period was 6 weeks. Food intake and weight gain in mice were compared (E and F). For the $3^{\text {rd }}$ study, we investigated the metabolic effects of genetic deletion of IL- 6 and IL-1 $\beta$ in $\mathrm{Ctns}^{-/}$mice beyond nutritional stimulation by

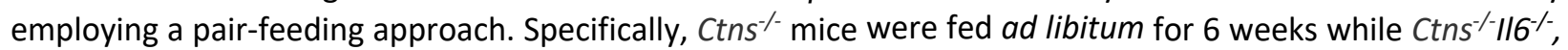
$\mathrm{Ctns}^{-/} \| 11 \beta^{-/}$and WT mice were pair-fed to that of $\mathrm{Ctns}^{-/}$mice (G). Weight gain, fat and lean content, 24-hr oxygen consumption, left gastrocnemius wet weight and in vivo muscle function (rotarod and grip strength) was measured in mice ( $\mathrm{H}$ to $\mathrm{N})$. Results of $2^{\text {nd }}$ and $3^{\text {rd }}$ study were expressed as mean $\pm \mathrm{SEM}$. Results of $\mathrm{Ctns}^{\%}$, $\mathrm{Ctns}^{-1} 116^{-/}$and $\mathrm{Ctns}^{-/} \| 11 \beta^{-/}$were compared to WT mice significance represented by \#). In addition, results of $\mathrm{Ctns}^{\%} / 16^{\%}$ and $\mathrm{Ctns}^{\%} / 11 \beta^{\%}$ mice were also compared to $\mathrm{Ctns}^{\%}$ mice, respectively (E to $\mathrm{N}$ ) and statistical significance ( $p$ value) is shown above bar.

Figure 2: Anakinra attenuates cachexia in $\mathrm{Ctns}^{\%}$ mice. Twelve-month old WT and $\mathrm{Ctns}^{\%}$ mice were treated with anakinra (2.5 mg.kg.day, IP) or normal saline as a vehicle control for 6 weeks. All mice were fed ad libitum for 6 weeks and food intake as well as weight were recorded (A \& B). In another experiment, to assess the beneficial effects of anakinra beyond its nutritional effects, we employed a pair-feeding strategy. Vehicletreated $\mathrm{Ctns}^{-/}$mice were given an ad libitum amount of food whereas other groups of mice were given an equivalent amount of food (C). Weight gain, fat and lean content, 24-hr oxygen consumption, left gastrocnemius wet weight and in vivo muscle function (rotarod and grip strength) was measured in the mice (D to J). Data are expressed as mean \pm SEM. Results of vehicle-treated $\mathrm{Ctns}^{-/}$mice were compared to vehicletreated WT mice while results of anakinra-treated $\mathrm{Ctns}^{-/}$mice were compared to that of anakinra-treated WT mice $(\# p<0.05)$. In addition, results of anakinra-treated $\mathrm{Ctns}^{-1-}$ mice were compared to vehicle-treated $\mathrm{Ctns}^{-/}$ mice and specific $p$ value is shown above bar.

Figure 3: Anakinra ameliorates energy homeostasis in adipose tissue and skeletal in $\mathrm{Ctns}^{-/}$mice. UCPs and ATP content in adipose tissue (inguinal white adipose tissue and brown adipose tissue) and gastrocnemius muscle were measured. Results are analyzed and expressed as in Figure 2.

Figure 4: Anakinra attenuates adipose tissue browning in $\mathrm{Ctns}^{-/}$mice. Gene expression of beige adipocyte markers (CD137, Tmem26 and Tbx-1) in inguinal white adipose tissue was measured. Gene expression of Cox2 signaling pathway (Cox2 and Pgf2 $\alpha$ ) and toll like receptor pathway (TIr2 and MyD88) in inguinal white adipose tissue was also measured. Gene expression was measured by qPCR. Final results were expressed in arbitrary units, with one unit being the mean level in vehicle-treated WT mice. Results are analyzed and expressed as in Figure 2.

Figure 5: Anakinra normalizes muscle fiber size and attenuates muscle fat infiltration in $\mathrm{Ctns}^{\%}$ mice. Representative photomicrographs of gastrocnemius with H\&E staining (A-D). Average gastrocnemius crosssectional area was measured (E). Visualization of quantification of fatty infiltration by Oil Red $O$ analysis in gastrocnemius muscle (F-J). Final results were expressed in arbitrary units, with one unit being the mean staining intensity in vehicle-treated WT mice. Difference among various groups of mice were analyzed as in Figure 2. 
bioRxiv preprint doi: https://doi.org/10.1101/2021.02.05.429989; this version posted February 7, 2021. The copyright holder for this preprint (which was not certified by peer review) is the author/funder. All rights reserved. No reuse allowed without permission.

Figure 6: Anakinra attenuates signaling pathways implicated in muscle wasting in $\mathrm{Ctns}^{\text {\%- }}$ mice. Gastrocnemius muscle relative phospho-Akt (pS473) / total Akt ratio, relative phospho-ERK 1/2 (Thr202/Tyr204) / total ERK 1/2 ratio, relative phospho-JNK (Thr183/Tyr185) / total JNK ratio, relative phospho-p38 MAPK (Thr180/Tyr182) / total p38 MAPK and relative phosphorylated NF-KB p65 (Ser536) / total p65 ratio as well as relative phosphor

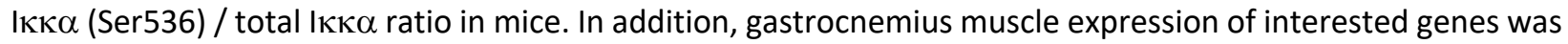
measured by qPCR. Final results were expressed in arbitrary units, with one unit being the mean level in vehicletreated WT mice. Results are analyzed and expressed as in Figure 2.

Figure 7: Summary of the beneficial effects of anakinra on cachexia, energy homeostasis, adipose tissue browning and muscle wasting in $\mathrm{Ctns}^{-/}$mice. 


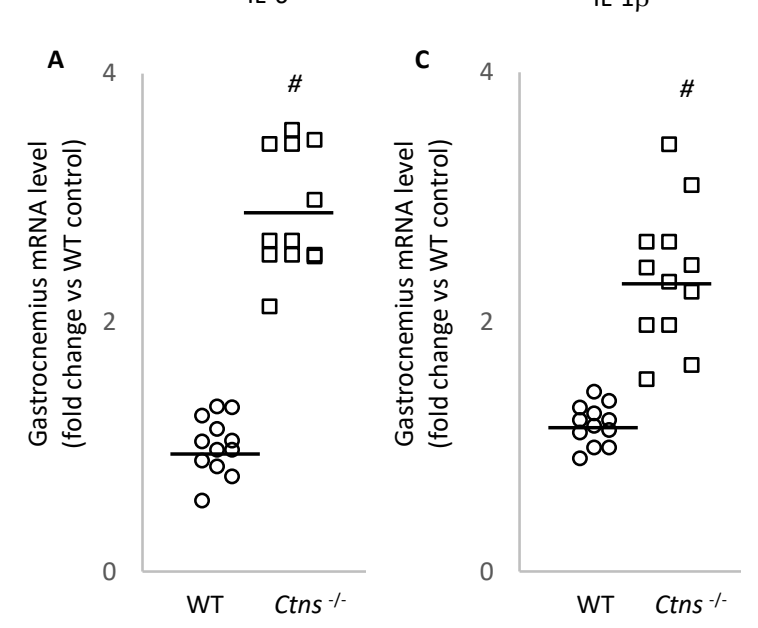

WT Ctns $\%$
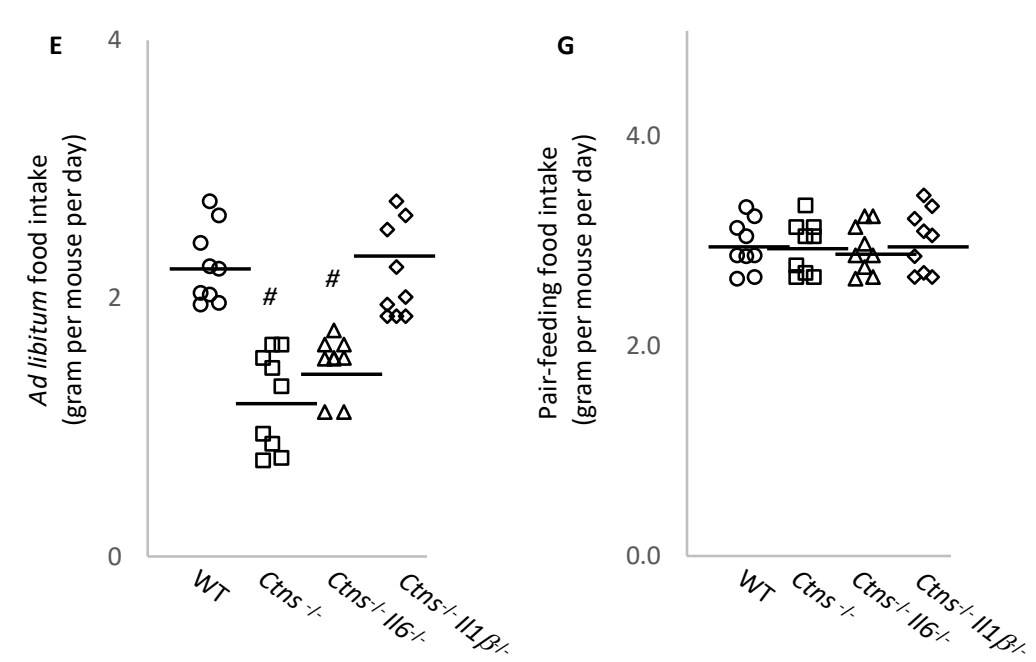

IL-1 $\beta$
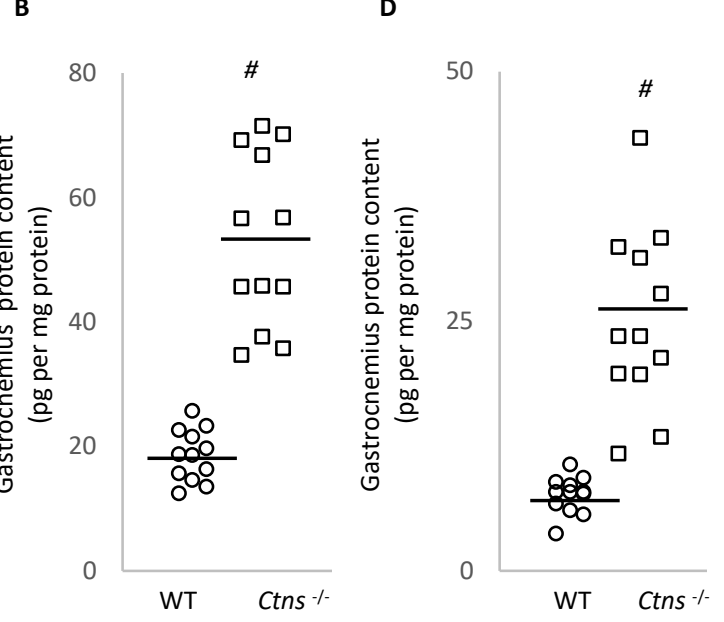

$\frac{p<0.05}{\overline{n . s}}$

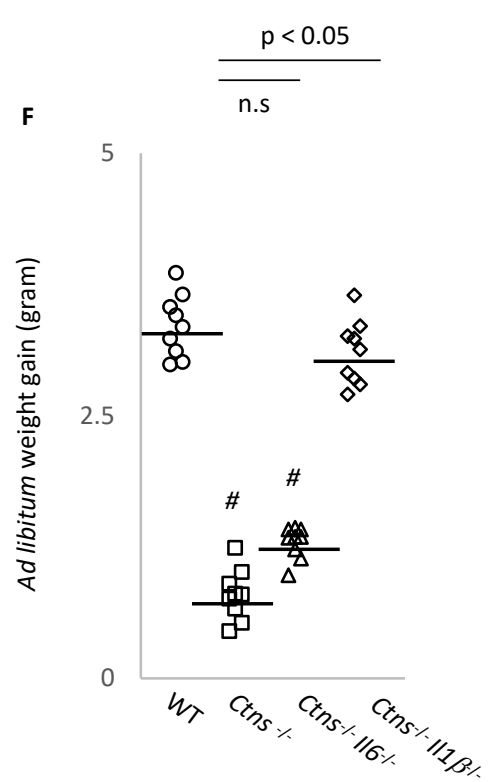

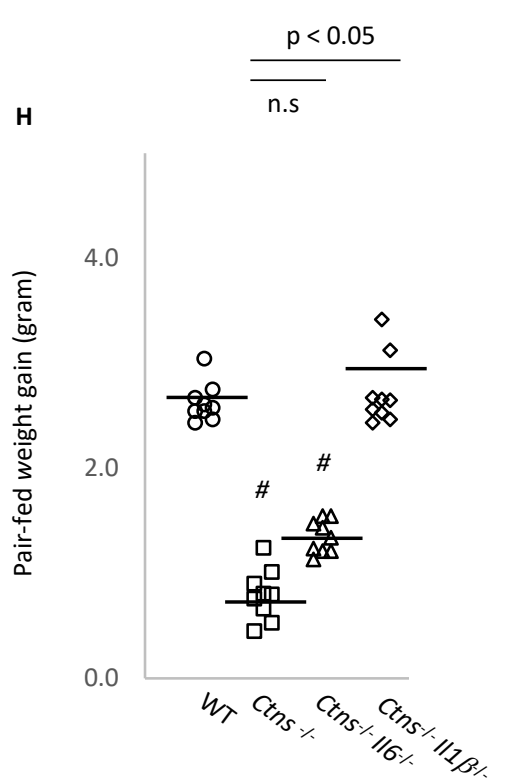

$\frac{p<0.05}{\overline{p<0.05}}$

$\frac{p<0.05}{\overline{n . s}}$

$\frac{\mathrm{p}<0.05}{\overline{\text { n.s }}}$
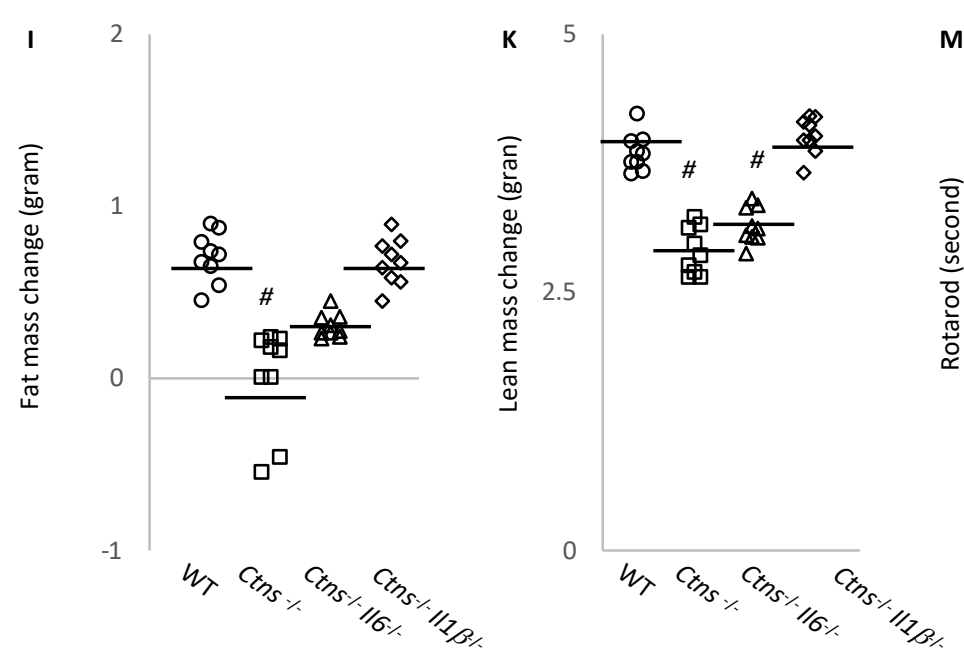

M

300

$\stackrel{\infty}{\circ}$

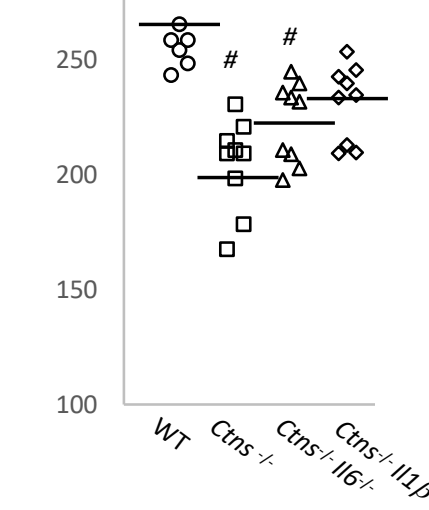

$\frac{p<0.05}{\#^{n . s}}$

$\mathrm{L}$

$\frac{p<0.05}{\overline{p<0.05}}$

$\frac{p<0.05}{\overline{n . s}}$

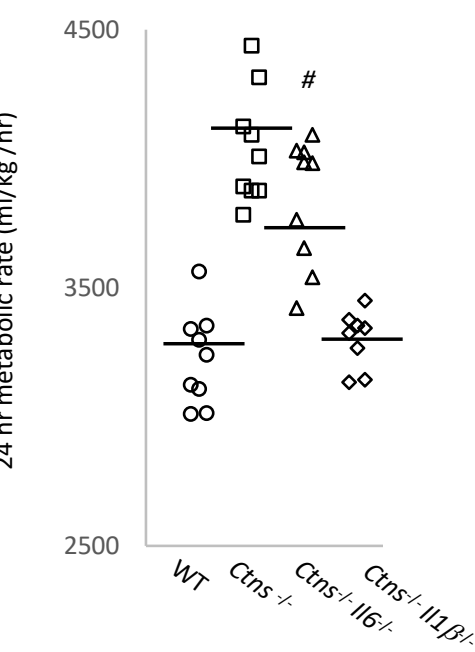

$\frac{8}{\infty 8}$

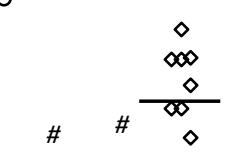

口 $\Delta \diamond$

$\Delta \frac{\Delta M}{\Delta}$

$\lim _{\rightarrow \Delta}^{\Delta}$

$\square^{\square}$

10

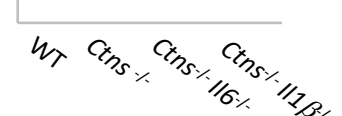



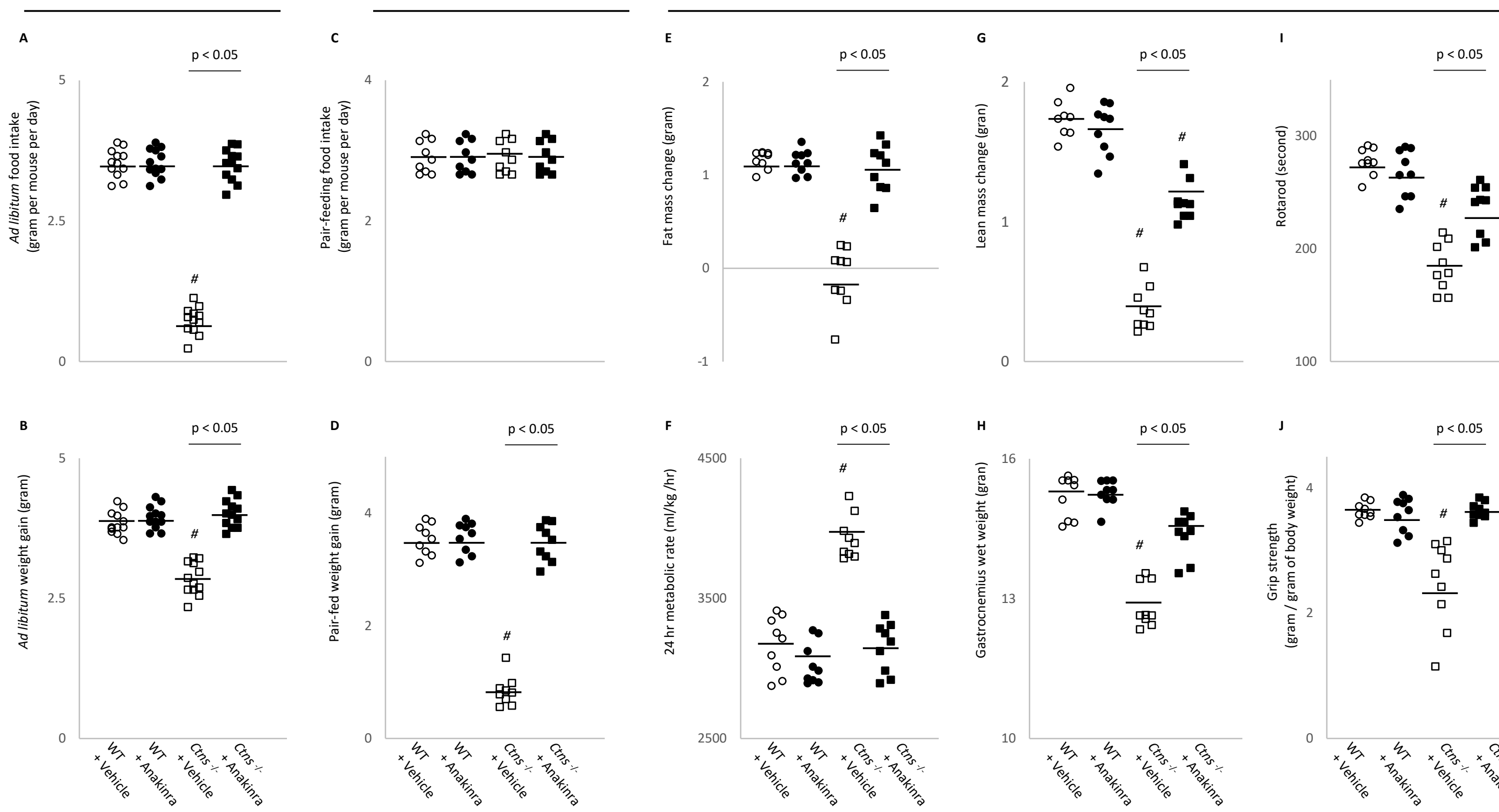

$p<0.05$
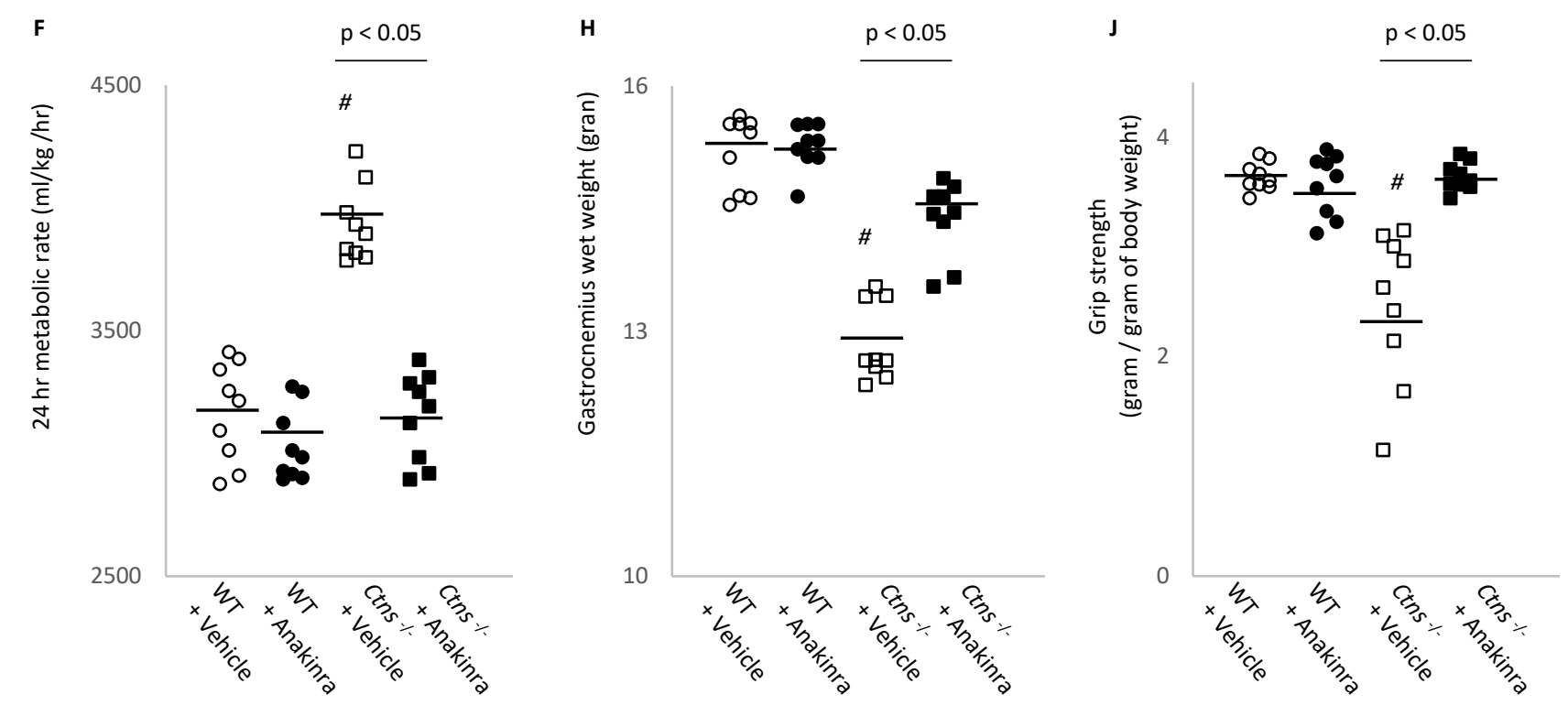
Figure 3
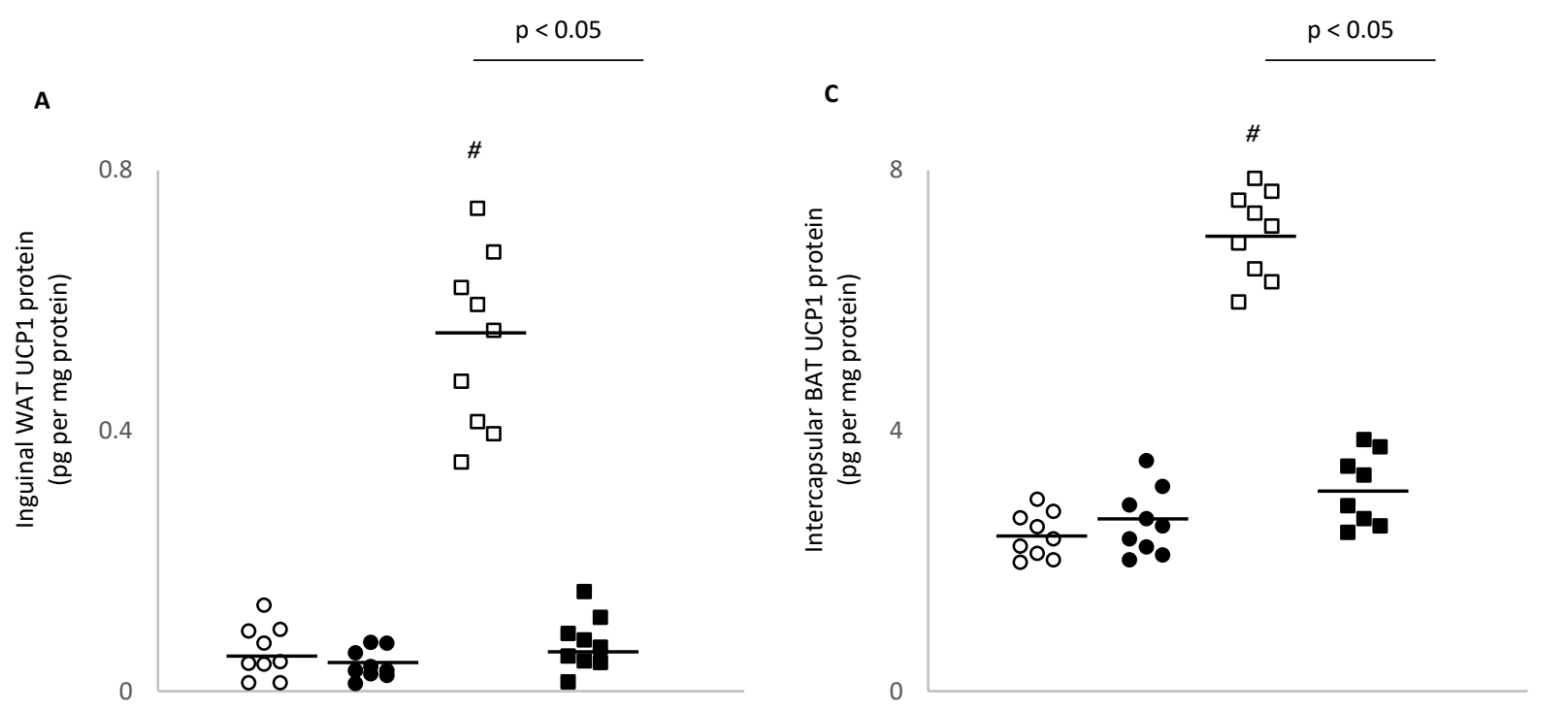

$p<0.05$
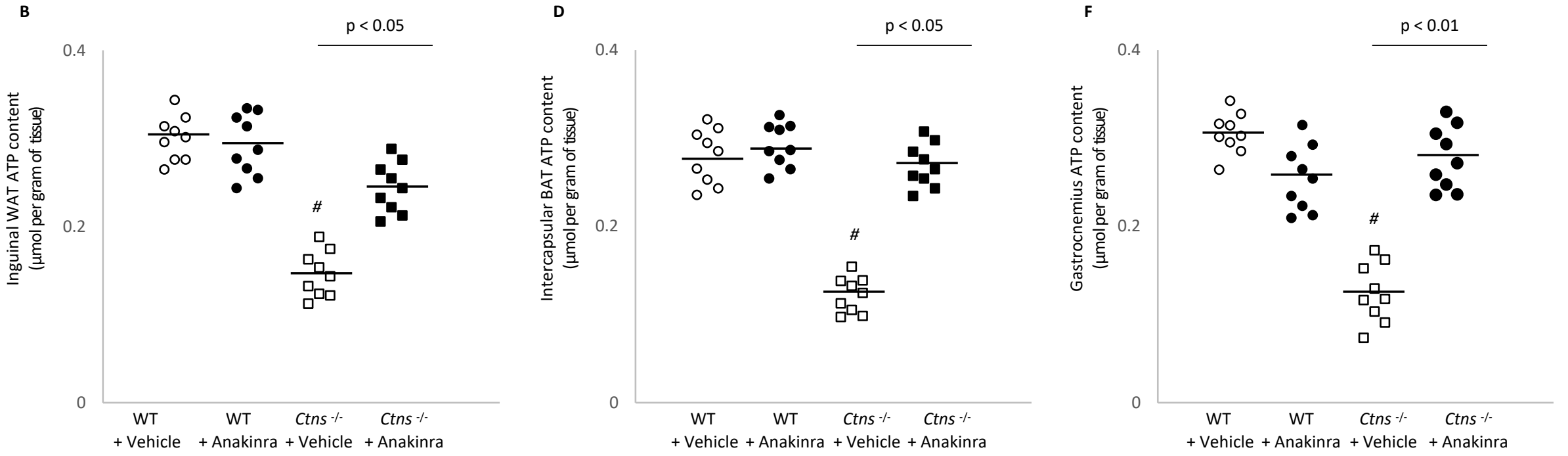
Figure 4

Inguinal WAT, beige adipocyte markers
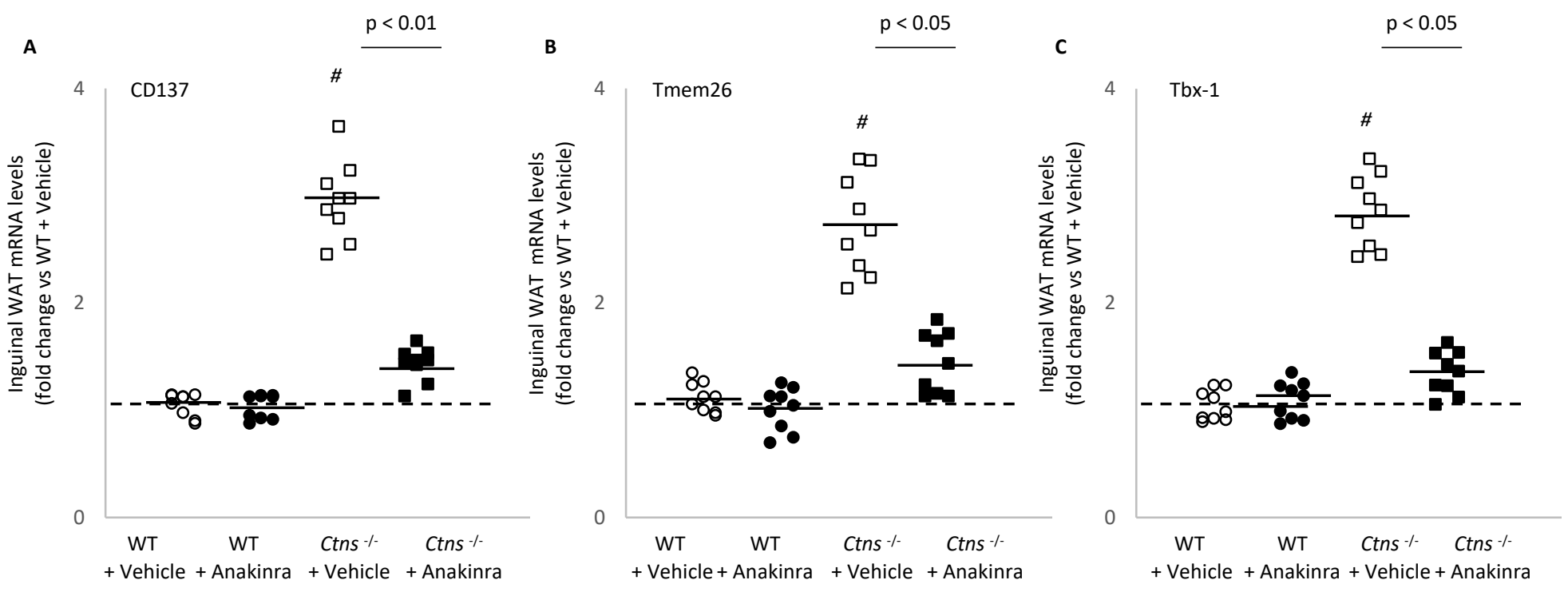

Key molecules implicated in WAT browning

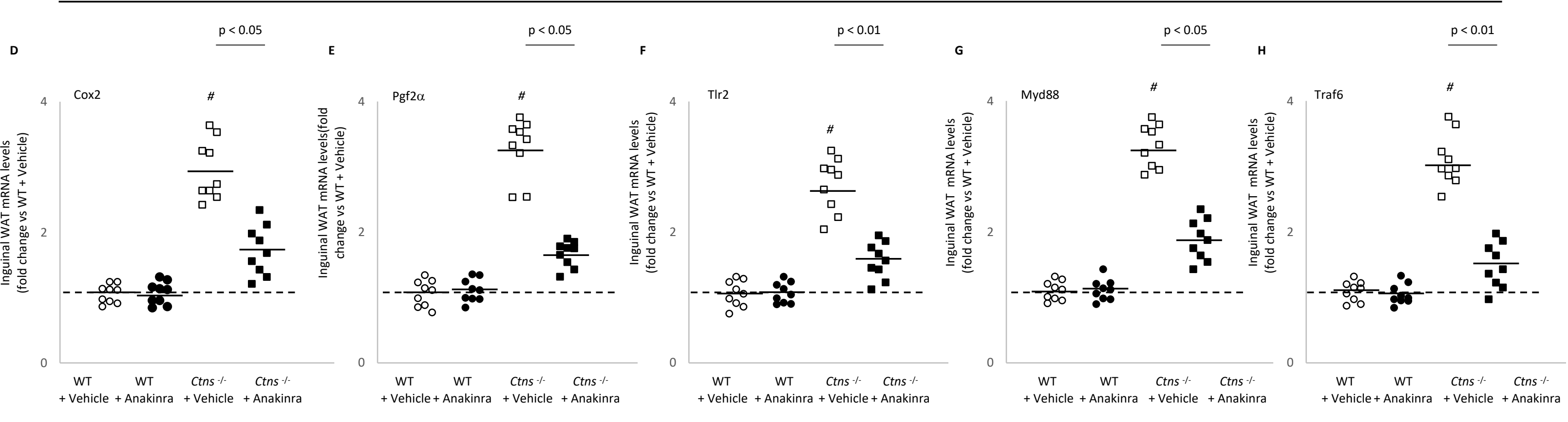



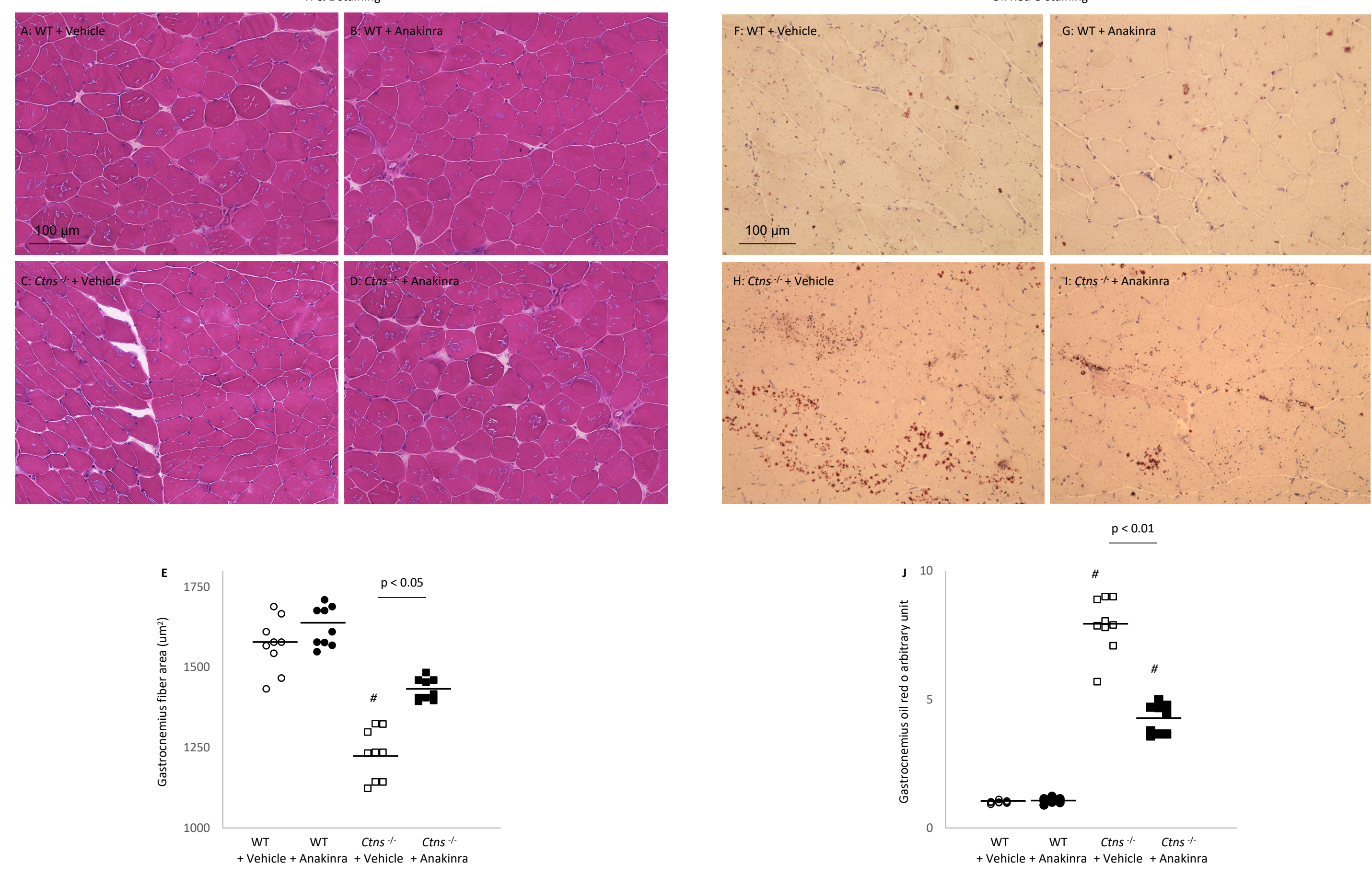


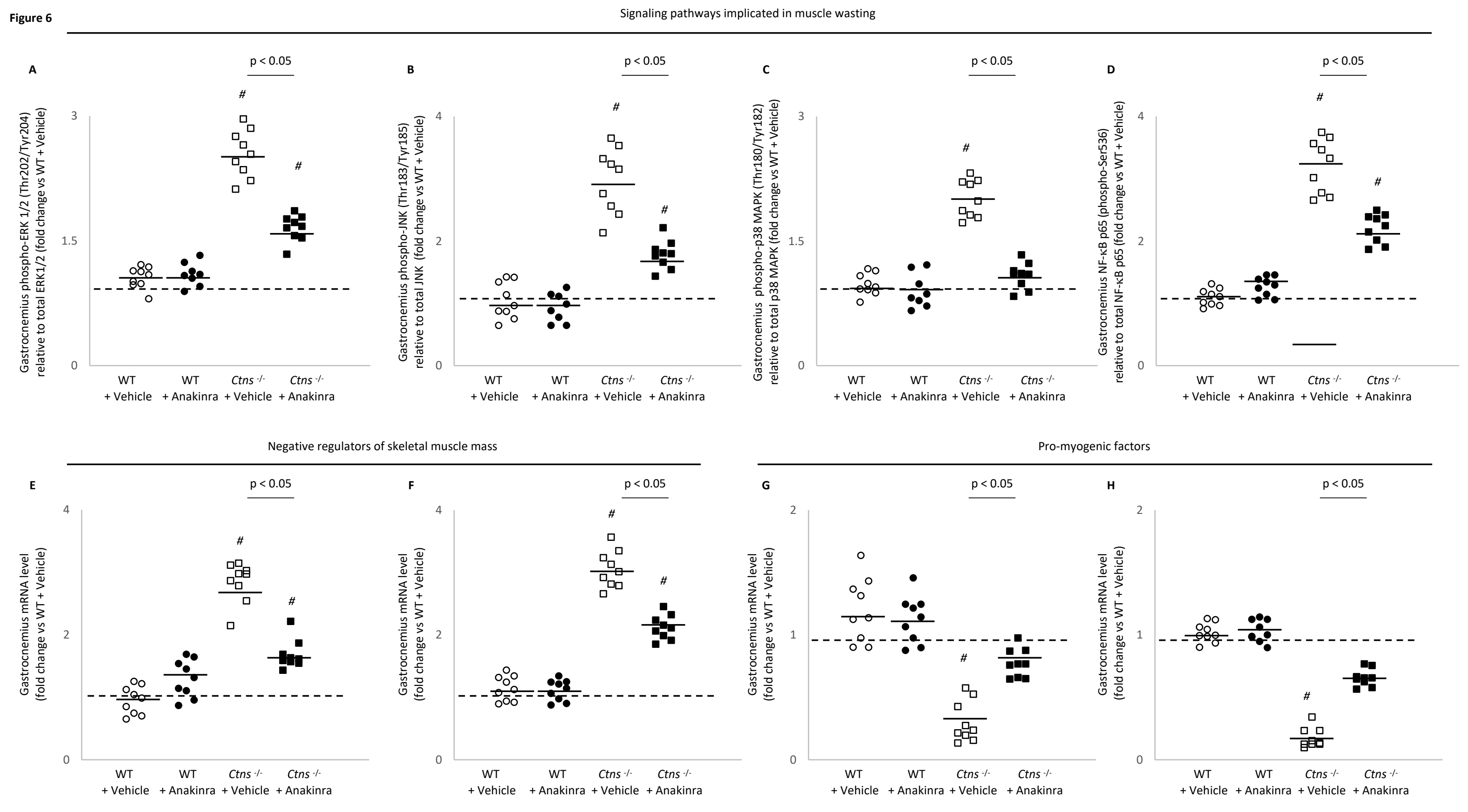


Results based on targeted investigation

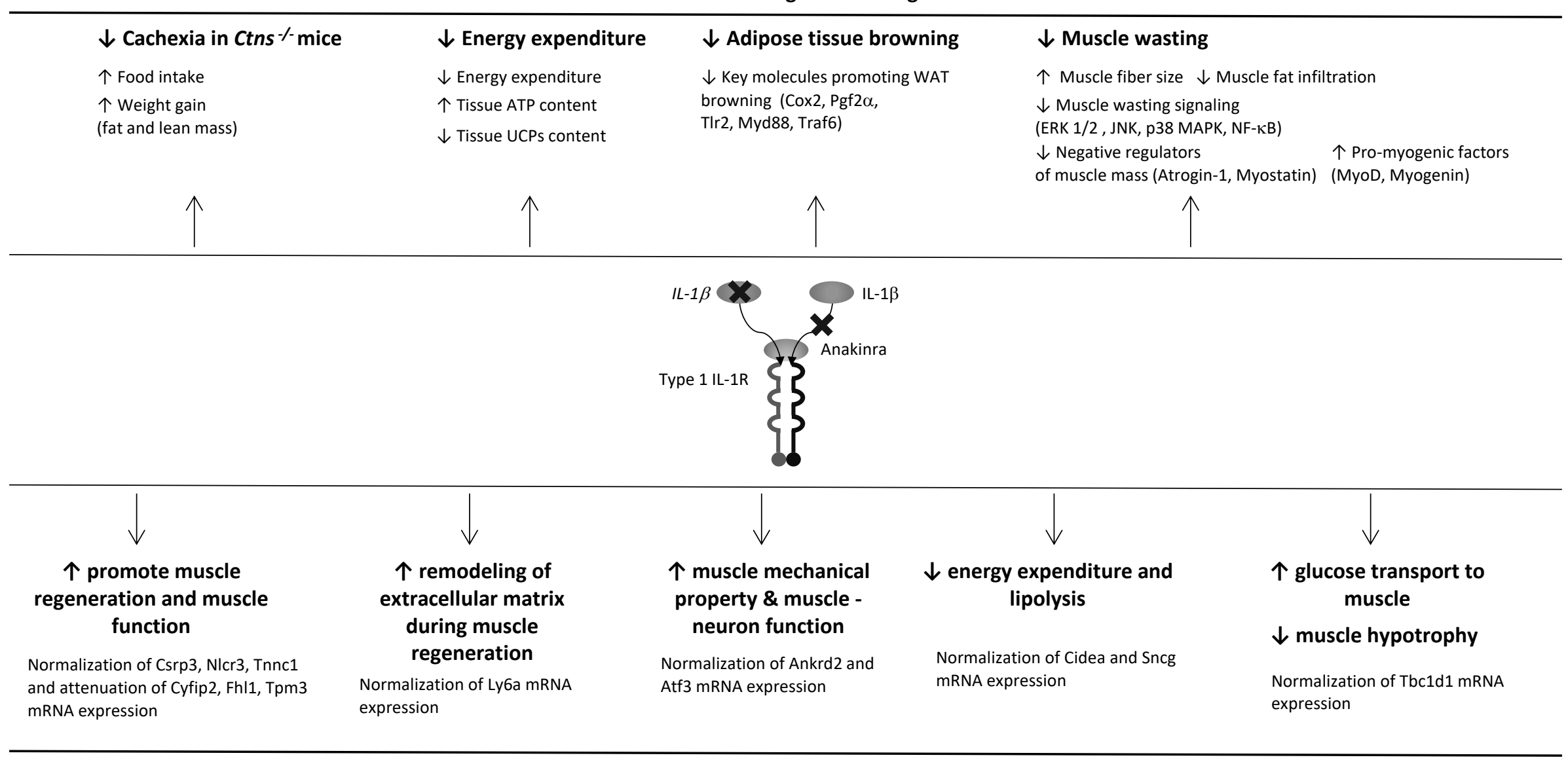

Results based on muscle RNAseq data 


\section{Supplemental information}

\section{Targeting interleukin-1 for reversing fat browning and muscle wasting in infantile nephropathic cystinosis}

Wai W Cheung ${ }^{1 *}$, Sheng $\mathrm{Hao}^{2^{*}}$, Ronghao Zheng ${ }^{3}$, Zhen Wang ${ }^{4}$, Alex Gonzalez ${ }^{1}$, Ping Zhou ${ }^{5}$, Hal M Hoffman ${ }^{6}$, Robert $\mathrm{H} \mathrm{Mak}^{1}$

${ }^{1}$ Pediatric Nephrology, Rady Children's Hospital San Diego, University of California, San Diego

${ }^{2}$ Department of Nephrology and Rheumatology, Shanghai Children's Hospital,

Shanghai Jiaotong University, China

${ }^{3}$ Department of Pediatric Nephrology, Rheumatology, and Immunology, Maternal and Child Health Hospital of Hubei Province, Tongji Medical College, Huazhong University of Science and Technology, China

${ }^{4}$ Department of Pediatrics, Shanghai General Hospital, Shanghai Jiaotong University, China

${ }^{5}$ Department of Pediatrics, the Second Affiliated Hospital of Harbin Medical University, China

${ }^{6}$ Division of Pediatric Allergy, Immunology, Rheumatology, Rady Children's Hospital San Diego University of California, San Diego

* These authors contributed equally to this work.

Correspondence:

Robert H Mak

Division of Pediatric Nephrology

Department of Pediatrics

University of California, San Diego

9500 Gilman Drive, MC0630, La Jolla, California 92093-0630

P: 858-822-6717

F: 858-822-6776

E-mail: romak@health.ucsd.edu 
Supplemental Table 1: Immunoassay information for blood and serum chemistry, muscle adenosine triphosphate content as well as muscle and adipose tissue protein analysis.

\begin{tabular}{|c|c|}
\hline Blood \& Serum chemistry & Assay information \\
\hline Bicarbonate \& BUN & VetScan Comprehensive Diagnostic Profile, Abaxix, 500-0038 \\
\hline Creatinine & LC-MS/MS method \\
\hline Muscle \& adipose tissue & Assay information \\
\hline ATP Assay Kit (Colorimetric / Fluorometric) & Abcam, ab83355 \\
\hline IL-1 $\beta$ & RayBiotech, ELM-IL1b-CL \\
\hline IL-6 & RayBiotech, ELM-IL6 \\
\hline $\begin{array}{l}\text { Mouse, rat, human ERK1/2, JNK, p38 MAPK } \\
\text { phosphorylation ELISA sampler kit }\end{array}$ & RayBiotech, CBEL-ERK-SK \\
\hline Mouse NFKB p65 (phospho-Ser536) ELISA kit & RayBiotech, PEL-NFKBP65-S536-T-1 \\
\hline Mouse total NFKB p65 ELISA kit & RayBiotech, PEL-NFKBP65-S536-T-1 \\
\hline Mouse Ucp1 ELISA kits & Aviva Systems Biology, OKCD02970 \\
\hline Mouse Ucp3 ELISA kits & Aviva Systems Biology, OKEH05259 \\
\hline
\end{tabular}


Supplemental Table 2S: PCR primer information.

\begin{tabular}{|c|c|c|}
\hline Gene & Forward primer sequence & $\underline{\text { Reverse primer sequence }}$ \\
\hline Ankrd2 & TGGACATGCTAGTGCTAGAGG & CGCTTTTTCTGCTTGCGTTTT \\
\hline Atf3 & GAGGATTTTGCTAACCTGACACC & TTGACGGTAACTGACTCCAGC \\
\hline Atrogin-1 & CAGCTTCGTGAGCGACCTC & GGCAGTCGAGAAGTCCAGTC \\
\hline Cidea & TGACATTCATGGGATTGCAGAC & GGCCAGTTGTGATGACTAAGAC \\
\hline CD137 & CGTGCAGAACTCCTGTGATAAC & GTCCACCTATGCTGGAGAAGG \\
\hline $\operatorname{Cox} 2$ & AACCCAGGGGATCGAGTGT & CGCAGCTCAGTGTTTGGGAT \\
\hline Csrp3 & GGGGGAGGTGCAAAATGTG & CAGGCCATGCAGTGGAAACA \\
\hline Cyfip2 & ATGACCACCCACGTCACTTTG & СCTGTCCTCGAAGTTCGTGTC \\
\hline Fhl1 & GACTGCCGCAAGCCCATAA & CCAAGGGGTGAAGGCACTT \\
\hline Fos & TTGAGCGATCATCCCGGTC & GCGTGAGTCCATACTGGCAAG \\
\hline IL-1 $\beta$ & GCAACTGTTCCTGAACTCAACT & АTCTTTTGGGGTCCGTCAACT \\
\hline IL-6 & TGGGGCTCTTCAAAAGCTCC & AGGAACTATCACCGGATCTTCAA \\
\hline Ly6a & AGGAGGCAGCAGTTATTGTGG & CGTTGACCTTAGTACCCAGGA \\
\hline Mup1 & GAAGCTAGTTCTACGGGAAGGA & AGGCCAGGATAATAGTATGCCA \\
\hline MyD88 & TCATGTTCTCCATACCCTTGGT & AAACTGCGAGTGGGGTCAG \\
\hline Myl2 & ATCGACAAGAATGACCTAAGGGA & ATTTTTTCACGTTCACTCGTCCT \\
\hline Myl3 & TGGGGAAGCCAAAACAGGAAG & AGCCATCAGTTTCTCTACCTCA \\
\hline MyoD & CCACTCCGGGACATAGACTTG & AAAAGCGCAGGTCTGGTGAG \\
\hline Myogenin & GAGACATCCCCCTATTTCTACCA & GCTCAGTCCGCTCATAGCC \\
\hline Myostatin & AGTGGATCTAAATGAGGGCAGT & GTTTCCAGGCGCAGCTTAC \\
\hline Nlrc3 & CAGATTGGTAACAAAGGAGCCA & CGTTCGGTTTATCTTCAGAGCA \\
\hline Pgf2 $\alpha$ synthase & CTGGACTCATCGCAAACACAA & AGGAAGCCTTTGACTTCTGTCTA \\
\hline Sell & TACATTGCCCAAAAGCCCTTAT & CATCGTTCCATTTCCCAGAGTC \\
\hline $\operatorname{Sln}$ & CTTGGGTGGATACCACGTCTG & СTTCTGTCTTACAGCCATAGCC \\
\hline Sncg & AAAGACCAAGCAGGGAGTAACG & GACCACGATGTTTTCAGCCTC \\
\hline Spp1 & AGGAAGAAGTAGGCATTTCTGGT & TCGGCTCTGCAATGTTGTCTG \\
\hline Tbc1d1 & TTCCTGGGGGTGAGTCTCAG & GCAGGGCATTACGGTAGGAG \\
\hline Tbx1 & CTGTGGGACGAGTTCAATCAG & TTGTCATCTACGGGCACAAAG \\
\hline Tlr2 & GCAAACGCTGTTCTGCTCAG & AGGCGTCTCCCTCTATTGTATT \\
\hline Tmem26 & TTCCTGTTGCATTCCCTGGTC & GCCGGAGAAAGCCATTTGT \\
\hline Tnnc1 & GCGGTAGAACAGTTGACAGAG & CCAGCTCCTTGGTGCTGAT \\
\hline Tnni1 & ATGCCGGAAGTTGAGAGGAAA & TCCGAGAGGTAACGCACCTT \\
\hline Tpm3 & ACCACCATCGAGGCGGTAA & CCCTTTCCTCCGCATCATCA \\
\hline Traf6 & AAAGCGAGAGATTCTTTCCCTG & ACTGGGGACAATTCACTAGAGC \\
\hline Gapdh (inte & AGGTCGGTGTGAACGGATTTG & TGTAGACCATGTAGTTGAGGTCA \\
\hline
\end{tabular}


Supplemental Table 3S: Serum and blood chemistry of 12-month old $\mathrm{Ctns}^{\%}$ and wild type control mice. Data are expressed as mean $\pm \mathrm{SEM}$. Result of serum chemistry of $\mathrm{Ctns}^{-/-}$mice were compared to WT mice. ${ }^{\#} \mathrm{p}<0.05$, significantly increased in $\mathrm{Ctns}^{-/}$mice relative to WT mice.

\begin{tabular}{|l|c|c|}
\hline & WT & \multicolumn{1}{c|}{ Ctns $^{-/}$} \\
\hline & $\mathrm{n}=12$ & $\mathrm{n}=12$ \\
\hline BUN (mg/dL) & $26.4 \pm 3.5$ & $56.7 \pm 11.3^{\#}$ \\
\hline serum creatinine (mg/dL) & $0.06 \pm 0.03$ & $0.21 \pm 0.05^{\#}$ \\
\hline Bicarbonate (mmol/L) & $27.3 \pm 2.5$ & $27.3 \pm 3.2$ \\
\hline
\end{tabular}




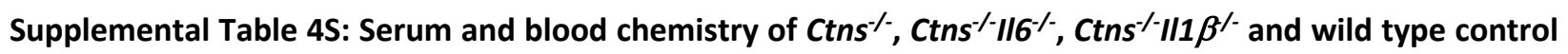
mice. Experiment was 6 weeks. Data are expressed as mean \pm SEM. Result of $\mathrm{Ctns} \mathrm{s}^{-/}, \mathrm{Ctns}^{-/ 116^{-/}}$and $\mathrm{Ctns}^{-/-111 \beta}$

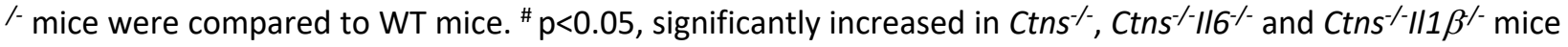
relative to WT mice.

\begin{tabular}{|l|c|c|c|c|}
\hline & WT & \multicolumn{2}{c|}{ Ctns $^{-/}$} & \multicolumn{2}{c|}{ Ctns $^{-/} \| 6^{-/}$} & \multicolumn{2}{c|}{ Ctns $^{-/} \| 1 \beta^{-/}$} \\
\hline BUN (mg/dL) & $\mathrm{n}=9$ & $\mathrm{n}=9$ & $\mathrm{n}=9$ & \multicolumn{1}{c|}{$\mathrm{n}=9$} \\
\hline serum creatinine (mg/dL) & $23.6 \pm 4.8$ & $49.7 \pm 4.3^{\#}$ & $69.1 \pm 7.7^{\#}$ & $49.5 \pm 3.5^{\#}$ \\
\hline Bicarbonate (mmol/L) & $0.06 \pm 0.01$ & $0.17 \pm 0.04^{\#}$ & $0.20 \pm 0.03^{\#}$ & $0.14 \pm 0.02^{\#}$ \\
\hline
\end{tabular}


Supplemental Table 5S: Serum and blood chemistry of $\mathrm{Ctns}^{-/-}$and wild type control mice. Twelve-month old WT and $\mathrm{Ctns}^{\%}$ - mice were treated with anakinra ( $2.5 \mathrm{mg} . \mathrm{kg}$ per day, IP) or vehicle (normal saline) for 6 weeks. Vehicle treated $\mathrm{Ctns}^{-/}$mice were fed ad libitum while other group of mice were pair-fed with the same amount of rodent diet as consumed by vehicle treated $\mathrm{Ctns}^{-/}$mice. Result of serum chemistry of $\mathrm{Ctns}^{-}-$+Vehicle mice were compared to WT+Vehicle mice while results of $\mathrm{Ctns}^{-/}+$Anakinra mice were compared to WT+Anakinra mice. Data are expressed as mean \pm SEM. ${ }^{\#}$ p $<0.05$, significantly increased in $\mathrm{Ctns}^{-/}+$Vehicle and $\mathrm{Ctns}^{-/}+$ Anakinra relative to $\mathrm{WT}+$ Vehicle and $\mathrm{WT}+$ Anakinra mice, respectively.

\begin{tabular}{|l|c|c|c|c|}
\hline & WT + Vehicle & WT + Anakinra & Ctns $^{-/}+$Vehicle & Ctns $^{-\%}+$ Anakinra \\
\hline BUN (mg/dL) & $\mathrm{n}=9$ & $\mathrm{n}=9$ & $\mathrm{n}=9$ & $\mathrm{n}=9$ \\
\hline serum creatinine (mg/dL) & $25.6 \pm 3.1$ & $24.7 \pm 3.7$ & $53.1 \pm 13.2^{\#}$ & $47.6 \pm 7.2^{\#}$ \\
\hline Bicarbonate (mmol/L) & $0.08 \pm 0.02$ & $0.09 \pm 0.03$ & $0.21 \pm 0.05^{\#}$ & $0.16 \pm 0.03^{\#}$ \\
\hline
\end{tabular}


Supplemental figure 1: Anakinra attenuates upregulated signature molecules implicated in $\mathrm{Ctns}^{-\%}$ associated muscle wasting and cachexia. Gastrocnemius muscle expression of interested genes in mice was measured by qPCR. Final results were expressed in arbitrary units, with one unit being the mean level in vehicle-treated WT mice. Data are expressed as mean \pm SEM. Results of vehicle-treated $\mathrm{Ctns}^{-1-}$ mice were compared to vehicletreated WT mice while results of anakinra-treated $\mathrm{Ctns}^{-/}$mice were compared to that of anakinra-treated WT mice. In addition, results of anakinratreated $\mathrm{Ctns}^{-1-}$ mice were compared to vehicle-treated $\mathrm{Ctns}^{-/}$mice. ${ }^{\#} \mathrm{p}<0.05$.

Supplemental figure 2: Anakinra attenuates downregulated signature molecules implicated in $\mathrm{Ctns}^{\%}$ associated muscle wasting and cachexia. Gastrocnemius muscle expression of interested genes in mice was measured by qPCR. Final results were expressed in arbitrary units, with one unit being the mean level in vehicle-treated WT mice. Data are expressed and analyzed as supplemental figure 1. 
Upregulated signature molecules implicated in Ctns $/$ associated muscle wasting and cachexia

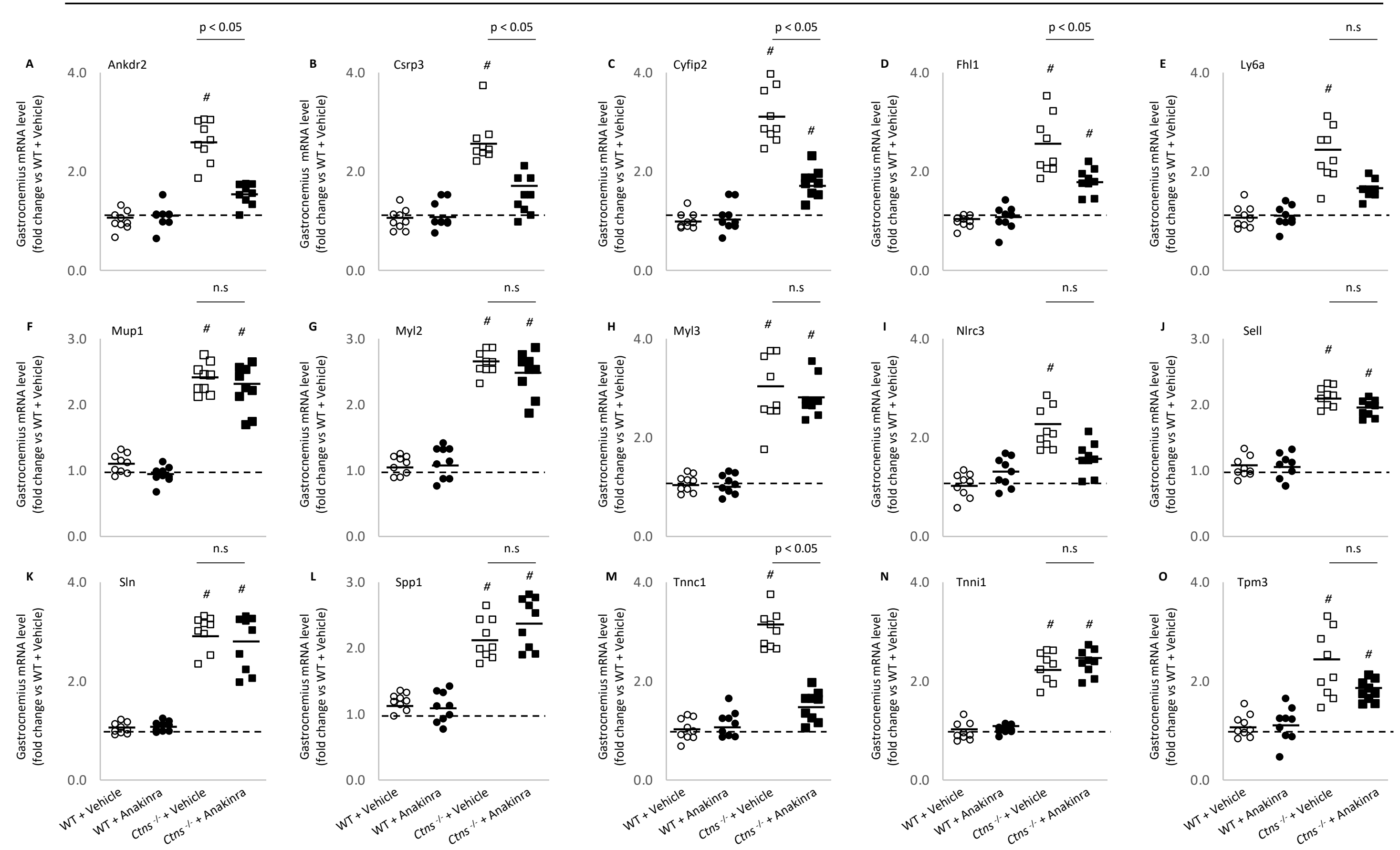




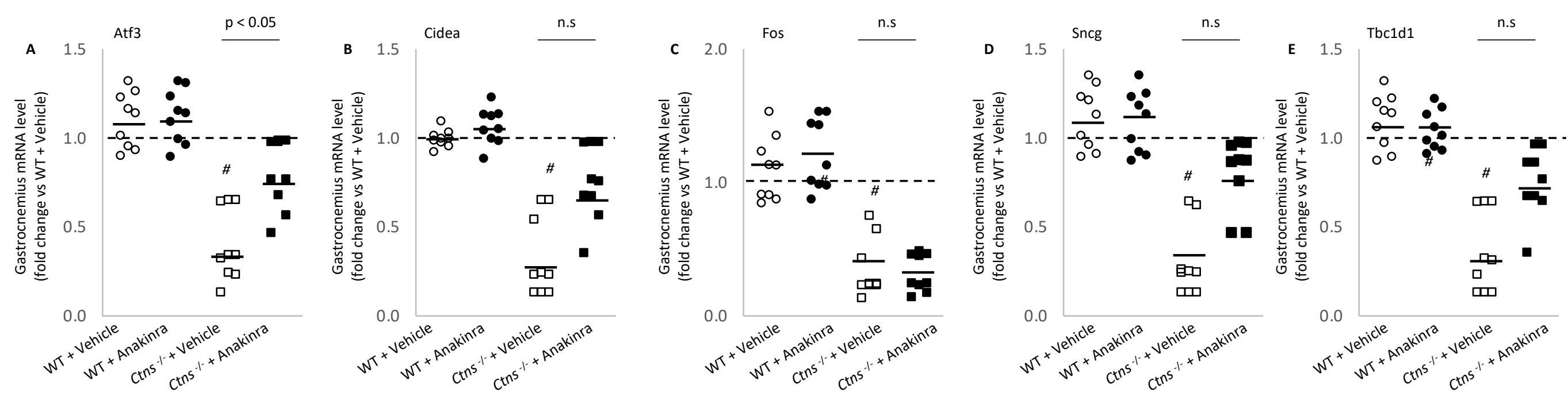

